Pacific

Journal of

Mathematics

BRANCHING IN THE UNIVERSAL COVER OF TAUT FOLIATIONS

SANDRA SHIELDS

Volume $203 \quad$ No. 1

March 2002 


\title{
BRANCHING IN THE UNIVERSAL COVER OF TAUT FOLIATIONS
}

\author{
SANDRA SHIELDS
}

\begin{abstract}
We study the topology of codimension one taut foliations of closed orientable 3-manifolds which are smooth along the leaves. In particular, we focus on the lifts of these foliations to the universal cover, specifically when any set of leaves corresponding to nonseparable points in the leaf space can be totally ordered. We use the structure of branching in the lifted foliation to find conditions that ensure two nonseparable leaves are left invariant under the same covering translation. We also determine when the set of leaves nonseparable from a given leaf is finite up to the action of covering translations. The hypotheses for the results are satisfied by all Anosov foliations.
\end{abstract}

\section{Introduction.}

This paper concerns codimension one foliations of closed orientable 3-manifolds where the leaves are $C^{1}$ submanifolds. We focus on the topological structure of the lifts of these foliations to the universal cover, particularly when the leaf space of the lifted foliation (i.e., the quotient space obtained by identifying points on the same leaf) is nonHausdorff.

When the lift of a foliation has a Hausdorff leaf space homeomorphic to $R$, the foliation is said to be " $R$-covered." Foliations with this property are particularly nice in the sense that they are completely determined by the action of the fundamental group of the manifold on the leaf space of the universal cover $[\mathbf{P a}]$. These foliations are well-understood and have served as a powerful tool, particularly in the study of Anosov flows. For example, if an Anosov foliation is $R$-covered, then the other Anosov foliation associated with the flow also has this property and the Anosov flow can be shown to be transitive [So, Ba1, Ba2]. Certain restrictions on the manifold in combination with the $R$-covered property have been used to show the Anosov flow is conjugate to a standard model; that is, a geodesic flow or a suspension of an Anosov diffeomorphism $[\mathbf{P l 1}, \mathbf{P l 2}, \mathbf{G h}]$. In addition, Fenley has used the $R$-covered hypothesis to uncover the rich structure of metric and homotopy properties in flow lines of many Anosov flows [Fe1, Fe2]. 
In contrast, foliations without the $R$-covered property are not generally well-understood. The nonHausdorff leaf space of the lifted foliation can, in some cases, be very complex [Im]. Consequently, little is known about the structure of these foliations, except in the Anosov case, and it is only recently that the structure of non R-covered Anosov foliations has been fully understood. (Examples include Anosov foliations tangent to a nontransitive Anosov flow, the first of which was constructed by Franks and Williams $[\mathbf{F r}-\mathbf{W i}]$, and the Anosov foliations tangent to the Bonatti and Langevin flow $[$ Bo-La $]$.)

In $[\mathbf{F e} 4]$, Fenley studies the lift of Anosov foliations to the universal cover and completely determines the structure of the set of "branching leaves" (i.e., leaves whose quotients in the leaf space are nonHausdorff points and which therefore correspond to positive or negative branching in the leaf space). He finds that this structure is very rigid and is strongly related to the topology of the ambient manifold below, the dynamics of the flow, and the metric behavior of the stable and unstable foliations. For example, Fenley shows that if two leaves $\widehat{A}$ and $\widehat{B}$ are nonseparable in the leaf space, then they are periodic; in fact, they cover leaves containing freely homotopic closed orbits of the Anosov flow, and $\widehat{A}$ and $\widehat{B}$ are left invariant under the same covering translation. This result is then used to show that, up to the action of covering translations, there are at most finitely many branching leaves. It is also a critical step in the proof that there is finite branching between the branching pair $(\widehat{A}, \widehat{B})$; that is, there are at most finitely many leaves "between" $\widehat{A}$ and $\widehat{B}$ which are nonseparable from $\widehat{A}$ and $\widehat{B}$. (A leaf $\widehat{C}$ in the stable Anosov foliation is said to be "between" leaves $\widehat{A}$ and $\widehat{B}$ if there is a leaf $\widehat{P}$ in the unstable Anosov foliation, through $\widehat{C}$, such that $\widehat{A}$ and $\widehat{B}$ lie on opposite sides of $\widehat{P}$.)

Here we shall consider the class of non $R$-covered taut foliations and show that by replacing the Anosov restriction with more general conditions we can ensure properties similar to those observed in Anosov foliations. To make sense of these properties in the more general setting, it will be necessary to develop a suitable definition of betweeness for nonseparable leaves in the universal cover. For this we use a flow which is transverse to the foliation, and betweeness will depend on the flow used. In the Anosov case, the definition of betweeness given here will coincide with the usual definition when the appropriate choice of transverse flow is made.

We ensure that for any 3 nonseparable leaves, precisely one of the leaves lies between the other two by requiring that every branching leaf in the universal cover contains a curve, called a "dividing curve," whose saturation by orbits of the transverse flow satisfies certain conditions. (These conditions are given in Definition 1.2 and are shown to be sufficient in Proposition 1.5.) 
Throughout, $\widehat{F}$ shall represent the lift of $F$ to the universal cover. Given a branching leaf $\widehat{A} \in \widehat{F}$, we will let $E_{-}(\widehat{A})$ denote the set consisting of $\widehat{A}$ and all leaves nonseparable from $\widehat{A}$ on the negative side.

The main results are as follows:

Theorem A. Let $F$ be a taut foliation with transverse flow $\phi$ such that every branching leaf of $\widehat{F}$ contains a dividing curve for $\widehat{\phi}$. Given a branching leaf $\widehat{A} \in \widehat{F}$, suppose $E_{-}(\widehat{A})$ is order isomorphic to a subset of the integers. If there exists a covering translation d fixing $\widehat{A}$ such that $d(\widehat{A})<d(\widehat{B})$ whenever $\widehat{B} \in E_{-}(\widehat{A})$ and $\widehat{A}<\widehat{B}$, then each leaf in $E_{-}(\widehat{A})$ is left invariant by $d$. In particular, the quotient in $F$ of each leaf in $E_{-}(\widehat{A})$ contains a loop freely homotopic to a loop in A.

Using [Fe4, Theorems B and C] we shall see that the hypotheses of Theorem A are satisfied by all Anosov foliations. As a corollary, we obtain $[\mathbf{F e} 4$, Theorem D] under more general conditions.

By Theorem A, the set $E_{-}(\widehat{A})$ is order isomorphic to a subset of $Z$ only in the case that every $\widehat{B} \in E_{-}(\widehat{A})$ is left invariant by certain covering translations fixing $\widehat{A}$. We shall give necessary and sufficient conditions on a branched surface constructed from $\widehat{F}$ to ensure that this is the case (Proposition 2.5).

In [Go-Sh] it was shown that a branched surface $W$ carrying a foliation exhibits a specific type of local behavior when there is a pair of planar nonseparable leaves in the universal cover; in particular, $W$ lifts to a branched surface $\widehat{W}$ in the universal cover and contains an arc, called a "branching arc", whose lift to $\widehat{W}$ links the images of the branching pair in $\widehat{W}$. A branching arc with restricted branchings along its interiors is said to be "simple". These definitions will be may more precise in Section 2 where we prove:

Theorem B. Let $F$ be a taut foliation with transverse flow $\phi$ such that every branching leaf of $\widehat{F}$ contains a dividing curve for $\widehat{\phi}$. Given a branching leaf $\widehat{A} \in \widehat{F}$, suppose $E_{-}(\widehat{A})$ is order isomorphic to the integers. There exists a branched surface $W$ carrying $F$ such that some simple branching arc in $W$ has at least 3 lifts linking distinct branching pairs in $E_{-}(\widehat{A})$ if and only if there are at most finitely many leaves of $F$ that lift to a leaf in $E_{-}(\widehat{A})$; in other words, the set $E_{-}(\widehat{A})$ is finite up to the action of covering translations.

Recall that if $F$ is Anosov, then for every leaf $\widehat{A}$ in the universal cover the set $E_{-}(\widehat{A})$ is order isomorphic to a subset of the integers. So by [Fe4, Theorem F], the conditions in Theorem B are satisfied by all Anosov foliations.

As noted in [Fe4], the number of leaves between a branching pair is a measure of the complexity of the branching. For example, finiteness of branching is, in some sense, a characteristic of foliations with a simple branching structure. Here, we show the following: 
Theorem C. Let $F$ be a taut foliation with transverse flow $\phi$ such that each branching leaf of $\widehat{F}$ contains a dividing curve for $\widehat{\phi}$. For every leaf $\widehat{A}$ in $\widehat{F}$, the set $E_{-}(\widehat{A})$ is order isomorphic to a subset of the rational numbers $Q$.

We also use branch surfaces to obtain the following information about the structure of the branching between two nonseparable leaves:

Theorem D. Let $F$ be a taut foliation with transverse flow $\phi$ such that each branching leaf of $\widehat{F}$ contains a dividing curve for $\widehat{\phi}$. For any positively branching pair $\widehat{A}$ and $\widehat{B}$ in $\widehat{F}$, there is an order preserving map $f$ assigning each element of $E_{-}(\widehat{A})$ between $\widehat{A}$ and $\widehat{B}$ to a connected component of $[0,1]-$ $\Sigma, \Sigma$ a Cantor set, and such that $f(\widehat{A})=\{0\}$ and $f(\widehat{B})=\{1\}$. Moreover, for any finite subset $\left\{\widehat{C}_{1}, \ldots, \widehat{C}_{n}\right\}$ of leaves between $\widehat{A}$ and $\widehat{B}$, there is an imbedded copy $\widehat{\gamma}$ of $[0,1]$ in $\widehat{W}$ intersecting each $\widehat{\pi}\left(\widehat{C}_{i}\right)$ in $f\left(\widehat{C}_{i}\right)$ with the property that for every $\widehat{C^{\prime}} \in E_{-}(\widehat{A})$, either $\widehat{\pi}\left(\widehat{C^{\prime}}\right) \cap \widehat{\gamma}=\varnothing$ or $\widehat{\pi}\left(\widehat{C^{\prime}}\right) \cap \widehat{\gamma}=$ $f\left(\widehat{C^{\prime}}\right)$.

The author wishes to thank Annalisa Calini and Brenton LeMesurier for helpful conversations. She is also grateful to the referee for his careful reading of the manuscript and his comments which were very useful in improving this paper.

\section{Betweeness and finite branching.}

Throughout this paper, $F$ will be a $C^{0}$ codimension one taut foliation of a closed orientable Riemannian 3-manifold $M \neq S^{2} X S^{1}$ where the leaves are $C^{1}$ submanifolds. Passing to a double cover of $M$ if necessary, may assume that $F$ is transversely orientable. We let $\widehat{F}$ denote the lift of $F$ to the universal cover $\widehat{M}$. Likewise, for any leaf $L$ (curve $\gamma)$ in $M, \widehat{L}(\widehat{\gamma}$ respectively) will refer to one of its lifts to $\widehat{M}$.

Definition 1.1. Given two leaves $\widehat{A}$ and $\widehat{B}$ of $\widehat{F}$, we say $(\widehat{A}, \widehat{B})$ is a branching pair if the points in the leaf space of $\widehat{F}$ representing $\widehat{A}$ and $\widehat{B}$ respectively are nonseparable; that is, $\widehat{A}$ and $\widehat{B}$ do not have disjoint saturated neighborhoods. In this case $\widehat{A}(\widehat{B})$ corresponds to a nonHausdorff point in the leaf space and is called a branching leaf. We say the branching pair $(\widehat{A}, \widehat{B})$ is a positively (negatively) branching pair if $\widehat{A}$ and $\widehat{B}$ are nonseparable on their negative (respectively positive) sides; i.e., $\widehat{A}$ and $\widehat{B}$ correspond to positive (respectively negative) branching in the leaf space of $\widehat{F}$. When $\widehat{F}$ contains only finitely many branching leaves we say it has finite branching.

Recall that a nonsingular flow on a 3-manifold $M$ is "Anosov" if for some Riemannian metric on $M$ the tangent bundle to the manifold has a continuous flow-invariant splitting into a Whitney sum, $T M=E^{0} \oplus E^{u} \oplus E^{s}$, of the 
line bundle $E^{0}$ tangent to the flow, and two other bundles, $E^{u}$ and $E^{s}$, so that the flow is exponentially expanding in the direction of $E^{u}$ and exponentially contracting in the direction of $E^{s}$. Associated with each Anosov flow is a foliation of $M$, called the "stable (unstable)" Anosov foliation, which is tangent to the subbundle spanned by $E^{0}$ and $E^{s}$ ( $E^{0}$ and $E^{u}$ respectively) [An]. A leaf $\widehat{C}$ in the stable (unstable) Anosov foliation of $\widehat{M}$ that is nonseparable from leaves $\widehat{A}$ and $\widehat{B}$ is said to be "between" $\widehat{A}$ and $\widehat{B}$ if $\widehat{A}$ and $\widehat{B}$ lie on opposite sides of a leaf, through $\widehat{C}$, in the unstable (respectively stable) Anosov foliation. By $[\mathbf{F e} 4]$, there are at most finitely many leaves between any branching pair $(\widehat{A}, \widehat{B})$ which are nonseparable from $\widehat{A}$ and $\widehat{B}$.

Here we adopt a notion of betweeness for leaves in the universal cover $\widehat{M}$ which does not require a transverse foliation to $F$ in $M$. As in the Anosov case, we use a plane separating $\widehat{M}$ which consists of a saturation by orbits in a transverse flow $\widehat{\phi}$. Unlike the Anosov case, there is no natural choice for $\widehat{\phi}$ and betweeness here will depend on this choice of flow. However, when the foliation is stable (unstable) Anosov, choosing $\widehat{\phi}$ to be the flow in the unstable (stable respectively) direction will yield the usual notion of betweeness.

The results which follow concern the class of foliations with the property that for any 3 nonseparable leaves in the universal cover, precisely one of these leaves lies between the other two. We show that when there is finite branching between any branching pair $(\widehat{A}, \widehat{B})$ in $\widehat{F}$, certain nontrivial covering translations fixing $\widehat{A}$ will also leave $\widehat{B}$ invariant (Theorem 1.7). We then show that for any foliation in this class, each set of nonseparable leaves in $\widehat{F}$ is order isomorphic to a subset of the rational numbers $Q$ (Theorem 2.2) and use branched surfaces to describe the structure of this branching (Theorem 2.3).

The foliation $F$ is taut, so it is Reebless and therefore contains no vanishing cycles. Consequently, all leaves of $\widehat{F}$ are simply connected and, since we are assuming that $M \neq S^{2} X S^{1}, \widehat{F}$ is a foliation by planes of the universal cover $R^{3}$. So there exists a foliation $F_{0}$ of $R^{2}$ such that $\widehat{F}$ is topologically conjugate to the product $F_{0} X R([\mathbf{P a}],[\mathbf{G a}-\mathbf{K a}])$. For every $r \in R$, we let $F_{0}(r)$ denote the image in $\widehat{F}$ of $F_{0} X\{r\}$ under the conjugacy map and $P_{0}(r)$ denote the imbedded copy of $R^{2}$ foliated by $F_{0}(r)$.

At any point $\widehat{x} \in P_{0}(r)$ we may use the transverse orientation to $\widehat{F}(r)$ as the first part of an orientation frame for $T \widehat{M}$, and a vector both tangent to $\widehat{F}$ and transverse to $P_{0}(r)$ in the direction of increasing $r$ as the second part of the frame. The third vector of the frame can be chosen tangent to the leaf of $F_{0}(r)$ through $\widehat{x}$ and used to define an orientation for this leaf. Throughout this paper, we assume that for any $r \in R$ the leaves of $F_{0}(r)$ are oriented in this manner. 
Let $\widehat{\chi}$ be a curve in a leaf of $\widehat{F}$. Given a nonsingular flow $\phi$ transverse to $F$, let $\widehat{\phi}$ denote its lift to the universal cover, and let $\widehat{\phi}(\widehat{\chi})$ represent the saturation of orbits through $\widehat{\chi}$. Since $F$ is Reebless, no orbit of $\widehat{\phi}$ can meet $\widehat{\chi}$ more than once. So if $\widehat{\chi}$ is a properly imbedded copy of $R$, then $\widehat{\phi}(\widehat{\chi})$ is a 2-manifold. It is also worth noting that $\widehat{\phi}(\widehat{\chi})$ is nowhere tangent to $\widehat{F}$.

Now suppose that for some $r$, the intersection of a leaf $\widehat{K}$ in $F_{0}(r)$ with $\widehat{\phi}(\widehat{\chi})$ is contained in some finite arc $\gamma$ in $\widehat{K}$ whose endpoints do not lie in $\widehat{\phi}(\widehat{\chi})$. We say $\widehat{K}$ and $\widehat{\phi}(\widehat{\chi})$ have "intersection number equal to 1 " if the ends of $\gamma$ lie on opposite sides of $\widehat{\phi}(\widehat{\chi})$. In other words, the intersection number of $\widehat{K}$ with $\widehat{\phi}(\widehat{\chi})$ is equal to 1 if all points in $\widehat{\phi}(\widehat{\chi}) \cap \widehat{K}$ lie between two points $x$ and $y$ in $\widehat{K}$, where $x$ and $y$ are on opposite sides of $\widehat{\phi}(\widehat{\chi})$.

Definition 1.2. A curve $\widehat{\chi}$ in a branching leaf $\widehat{C}$ of $\widehat{F}$ is a dividing curve for $\widehat{\phi}$ if the following conditions are satisfied:

(1) $\widehat{\phi}(\widehat{\chi})$ is a properly imbedded plane and

(2) there exist an $r_{\chi} \in R$ such that for almost every $r \geq r_{\chi}$, any leaf $\widehat{K}$ of $F_{0}(r)$ sufficiently close to $\widehat{C} \cap P_{0}(r)$ has intersection number with $\widehat{\phi}(\widehat{\chi})$ equal to 1.

When $\widehat{F}$ is a stable Anosov foliation, the orbit space, obtained by collapsing each orbit of the Anosov flow $\widehat{\sigma}$ to a point, is homeomorphic to $R^{2}$ [Fe1]. In this case, we may choose $P_{0}(0)$ to be an imbedded copy of this orbit space which is transverse to $\widehat{\sigma}$ and let $P_{0}(r)=\left\{\widehat{\sigma}(x, r): x \in P_{0}(0)\right\}$ for every $r \in R$. If we then choose $\widehat{\phi}$ to be the flow in the unstable direction $E^{u}$, we ensure that any branching leaf $\widehat{C}$ contains a dividing curve for $\widehat{\phi}$. To see this we first note that by $[\mathbf{F e} 4$, Theorem B] the quotient leaf $C$ in $M$ contains a closed orbit $\chi$ of the Anosov flow. For some lift $\widehat{\chi}$ of $\chi$ to $\widehat{C}$, the surface $\widehat{\phi}(\widehat{\chi})$ is a leaf in the unstable Anosov foliation, so $\widehat{\chi}$ satisfies Condition (1) above and is nowhere tangent to $P_{0}(r)$ for any $r \in R$. In fact, for every $r \in R, \widehat{\phi}(\widehat{\chi}) \cap P_{0}(r)$ is a leaf in the restriction of the unstable Anosov foliation to $P_{0}(r)$. So $\hat{\chi}$ also satisfies Condition (2) above.

We note that Condition (2) is not satisfied by finite depth foliations. It is also worth noting that Condition (1) in Definition 1.2 is fundamental to ensuring that $\widehat{\phi}(\widehat{\chi})$ separates $\widehat{M}$ into two disjoint open sets.

We now find alternative conditions which also guarantee Condition (1) is satisfied. In particular, we show the following:

Proposition 1.3. Let $\widehat{\chi}$ be a properly imbedded curve in a branching leaf of $\widehat{F}$ such that $\widehat{\phi}(\widehat{\chi})$ is an imbedded plane without boundary. If the induced foliation of $\widehat{\phi}(\hat{\chi})$ has finite branching (i.e., if there are finitely many leaves of $\widehat{F} \cap \widehat{\phi}(\widehat{\chi})$ which correspond to nonseparable points in the leaf space of the induced foliation) and if there is a flow on $\widehat{M}$ transverse to $\widehat{\phi}(\widehat{\chi})$ which is 
nowhere tangent to $\widehat{\phi}$, then $\widehat{\chi}$ satisfies Condition (1) for a dividing curve; that is, $\widehat{\phi}(\widehat{\chi})$ is a plane separating $\widehat{M}$ into two open components.

Proof. It is sufficient to show that if points in $\widehat{\phi}(\widehat{\chi})$ accumulate on some point $\widehat{p}$, then these points (together with $\widehat{p}$ ) lie on a finite curve in $\widehat{\phi}(\widehat{\chi})$. So suppose this is not the case. Since $\widehat{\phi}(\widehat{\chi})$ is an imbedded plane without boundary which is nowhere tangent to $\widehat{F}$, each leaf in the induced foliation of $\widehat{\phi}(\widehat{\chi})$ is an imbedded copy of $R$ without boundary; in particular, each component of $\widehat{\phi}(\widehat{\chi}) \cap \widehat{L}$ has this property, where $\widehat{L}$ is the leaf of $\widehat{F}$ containing $\widehat{p}$.

We first consider the case where some component of $\widehat{\phi}(\widehat{\chi}) \cap \widehat{L}$ is not properly imbedded in $\widehat{L}$. It then follows that $\widehat{\phi}(\widehat{\chi}) \cap \widehat{L}$ limits on a curve in $\widehat{L}$ which, by the existence of the flow $\widehat{\psi}$ transverse to $\widehat{\phi}(\widehat{\chi})$, is an embedded loop. (For example, $\widehat{\phi}(\widehat{\chi}) \cap \widehat{L}$ cannot double back near itself with opposite orientation as in Figure 1.1. In addition, $\widehat{\phi}(\widehat{\chi}) \cap \widehat{L}$ cannot be fractal for the same reason.) This loop in $\widehat{L}$ bounds an open disk $\widehat{D}$ in $\widehat{L}$ which is not met by the limiting component of $\widehat{\phi}(\widehat{\chi}) \cap \widehat{L}$. With the appropriate choice of metric, $\widehat{\phi}$ is everywhere perpendicular to $\widehat{L}$ and, by assumption, $\widehat{\psi}$ is nowhere tangent to $\widehat{\phi}$. So at each point in $\widehat{L}$ the tangent vector to $\widehat{\psi}$ projects orthogonally onto a nonzero vector in $\widehat{L}$. Since the Euler characteristic of $\widehat{D}$ is one, some integral curve of the resulting vector field is transverse to $\partial \widehat{D}$. Furthermore, the curve $\widehat{\phi}(\widehat{\chi}) \cap \widehat{L}$ is transverse to this vector field on $\widehat{L}$ and limits on $\partial \widehat{D}$. So there exists a disk $D^{\prime \wedge}$ in $\widehat{L}$ and containing $\widehat{D}$ whose boundary is everywhere transverse to the nonzero vector field on $\widehat{L}$ (see Figure 1.2). This is a contradiction since the Euler characteristic of $\widehat{D^{\prime}}$ is one.

The following cannot occur: 


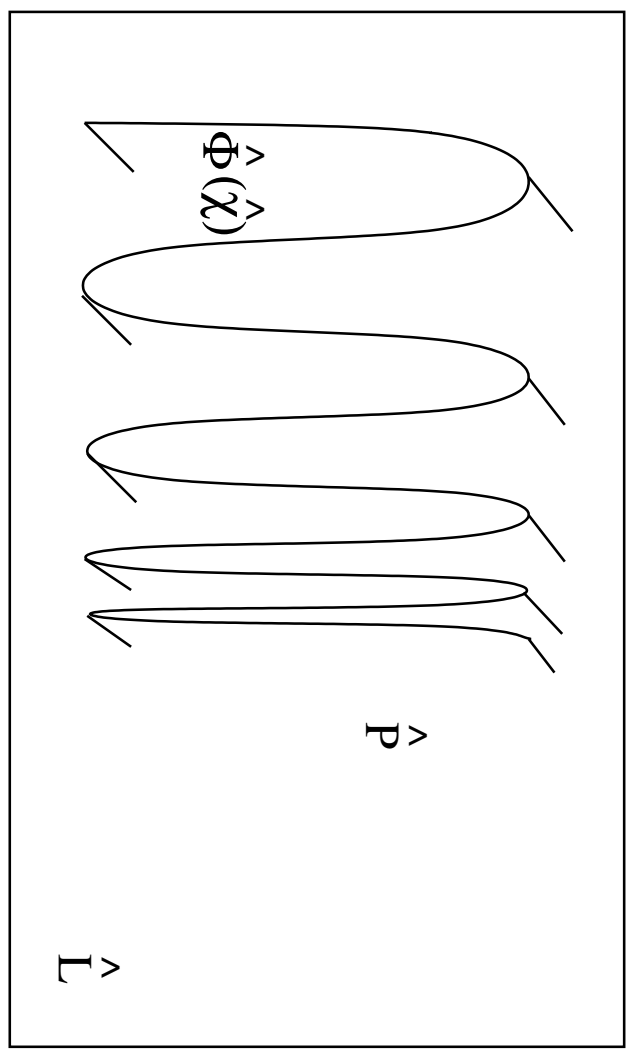

Figure 1.1.

The local picture is as follows, where the dashed lines indicate integral curves of the nonzero vector field on $\widehat{L}$ obtained by projecting $\widehat{\psi}$ : 


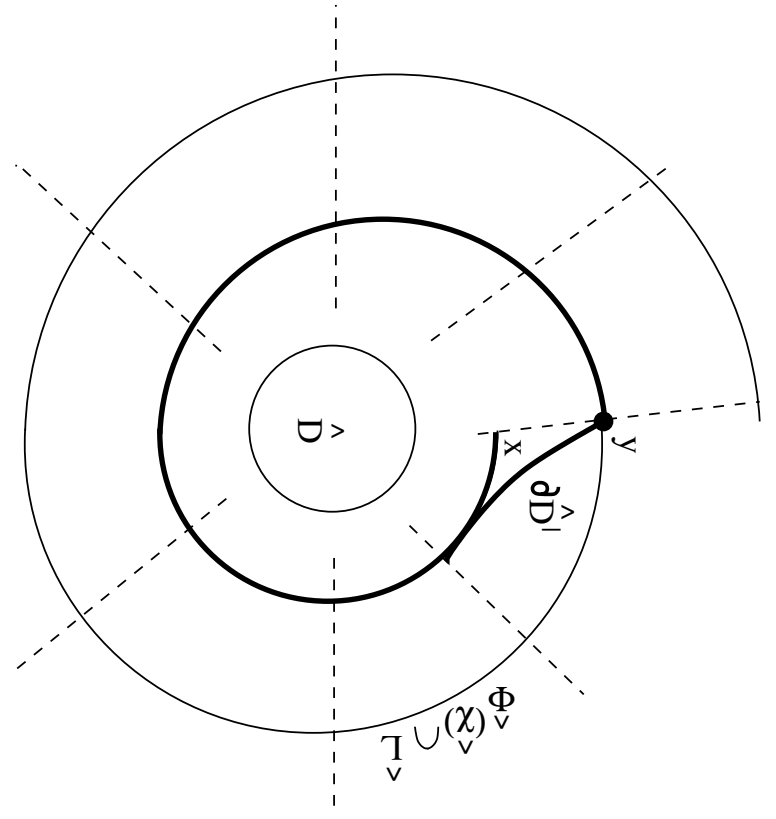

Figure 1.2. Modifying the composition of the integral curve from $x$ to $y$ with the curve in $\widehat{\phi}(\widehat{\chi}) \cap \widehat{L}$ from $y$ to $x$, we get a loop transverse to the integral curves.

Now suppose that the components of $\widehat{\phi}(\widehat{\chi}) \cap \widehat{L}$ are topologically closed in $\widehat{L}$. Since $\widehat{\phi}(\widehat{\chi})$ has no boundary and $\widehat{p}$ is an accumulation point of $\widehat{\phi}(\widehat{\chi})$, there is sequence $\left\{x_{i}\right\}$ of points in $\widehat{\phi}(\widehat{\chi}) \cap \widehat{L}$ limiting on $\widehat{p}$. Passing to a subsequence if necessary, we may assume that each $x_{i}$ is contained in a different component $E_{i}$ of $\widehat{\phi}(\widehat{\chi}) \cap \widehat{L}$ and that $\widehat{p} \notin E_{i}$ for all $i$. Now each $E_{i}$ is a leaf in the induced foliation of $\widehat{\phi}(\widehat{\chi})$. Since, by hypothesis, this foliation has finite branching, we may choose two such leaves $E_{i}$ and $E_{j}$ such that the corresponding points in the leaf space are joined by an imbedded Hausdorff 1-manifold. So there is a transverse arc from $E_{i}$ to $E_{j}$. We may modify the composition of this arc with a curve in $\widehat{L}$ from $E_{j}$ to $E_{i}$ in order to obtain a loop transverse to $\widehat{F}$, contradicting $F$ taut. (See Figure 1.3.)

Locally we have: 


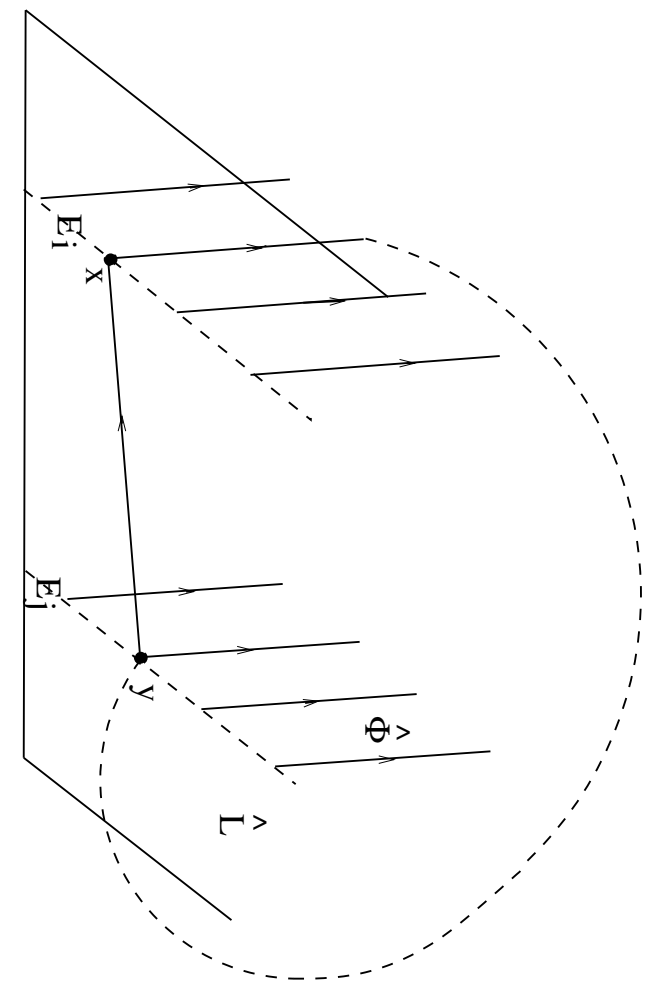

Figure 1.3. The dashed arc is transverse to $\widehat{F}$.

So $\widehat{\phi}(\widehat{\chi})$ is an imbedded plane.

It is worth noting that any 2 leaves connected by a transverse arc are separated from each other by any leaf intersecting the interior of the arc. It follows that if $\widehat{A}$ and $\widehat{B}$ are nonseparable and $\widehat{B}$ contains a dividing curve $\widehat{\beta}$ for $\widehat{\phi}$, then $\widehat{\phi}(\widehat{\beta})$ does not intersect $\widehat{A}$.

We now define betweeness for branching leaves as follows:

Definition 1.4. Given a branching pair $(\widehat{A}, \widehat{B})$ in $\widehat{F}$, suppose $\widehat{C}$ is a leaf which is nonseparable from $\widehat{A}$ and $\widehat{B}$. We say $\widehat{C}$ is between $\widehat{A}$ and $\widehat{B}$ with respect to the flow $\widehat{\phi}$ (and write $\widehat{A}-\widehat{C}-\widehat{B}$ ) if $\widehat{C}$ contains a dividing curve $\widehat{\chi}$ for $\widehat{\phi}$ such that $\widehat{A}$ and $\widehat{B}$ lie on opposite sides of $\widehat{\phi}(\widehat{\chi})$.

Note that "betweeness" is dependent on our choice of the transverse flow $\widehat{\phi}$. However, if $\widehat{F}$ is a stable (unstable) Anosov foliation and we choose $\widehat{\chi}$ to be the flow in the unstable (stable) direction, then this definition is equivalent to the usual definition ([Fe4]) of betweeness for branching leaves in an Anosov foliation.

Proposition 1.5. Given 3 nonseparable leaves $\widehat{A}, \widehat{B}$ and $\widehat{C}$ of $\widehat{F}$, if each contains a dividing curve, then precisely one of these leaves lies between the 
other two. Further, for dividing curves $\widehat{\chi}$ and $\widehat{\chi^{\prime}}$ in $\widehat{C}$, the leaves $\widehat{A}$ and $\widehat{B}$ are on opposite sides of $\widehat{\phi}(\widehat{\chi})$ if and only if $\widehat{A}$ and $\widehat{B}$ are on opposite sides of $\widehat{\phi}\left(\widehat{\chi^{\prime}}\right)$.

Proof. By hypothesis, $\widehat{A}, \widehat{B}$ and $\widehat{C}$ contain dividing curves $\widehat{\alpha}, \widehat{\beta}$ and $\widehat{\chi}$ respectively. By Condition (2) for a dividing curve, there exists an $r_{\alpha} \in R$, such that for almost every $r \geq r_{\alpha}$, any leaf $\widehat{K}$ of $F_{0}(r)$ sufficiently close to $\widehat{A} \cap P_{0}(r)$ has intersection number with $\widehat{\phi}(\widehat{\alpha})$ equal to 1 . Further, there exists $r_{\beta}$ and $r_{\chi}$ with the analogous property. Consequently, we may choose $r>\max \left\{r_{\alpha}, r_{\beta}, r_{\chi}\right\}$ so that any leaf $\widehat{K}$ of $F_{0}(r)$ chosen sufficiently close to $\widehat{A} \cap P_{0}(r), \widehat{B} \cap P_{0}(r)$ and $\widehat{C} \cap P_{0}(r)$ has intersection number with each of $\widehat{\phi}(\widehat{\alpha}), \widehat{\phi}(\widehat{\beta})$ and $\widehat{\phi}(\widehat{\chi})$ equal to 1 . Such a leaf $\widehat{K}$ contains a finite arc $\gamma$ whose endpoints $\gamma(0)$ and $\gamma(1)$ lie on opposite sides of $\widehat{\phi}(\widehat{\alpha}), \widehat{\phi}(\widehat{\beta})$ and $\widehat{\phi}(\widehat{\chi})$. Let $x(y)$ be the first (last respectively) point along $\gamma$ meeting $\widehat{\phi}(\widehat{\alpha}) \cup \widehat{\phi}(\widehat{\beta}) \cup \widehat{\phi}(\widehat{\chi})$. Since $\widehat{\phi}(\widehat{\alpha}), \widehat{\phi}(\widehat{\beta})$ and $\widehat{\phi}(\widehat{\chi})$ are pairwise disjoint, one of these planes contains neither $x$ nor $y$. Furthermore, $x$ and $y$ lie on opposite sides of this plane since $x(y)$ is on the same side as $\gamma(0)(\gamma(1)$ respectively). It follows that two of the planes $\widehat{\phi}(\widehat{\alpha}), \widehat{\phi}(\widehat{\beta})$ and $\widehat{\phi}(\widehat{\chi})$ lie on opposite sides of the third. In other words, precisely one of the leaves $\widehat{A}, \widehat{B}$ and $\widehat{C}$ lies between the other two.

Now suppose that $\widehat{A}$ and $\widehat{B}$ are on opposite sides of $\widehat{\phi}(\widehat{\chi})$, and let $\widehat{\chi^{\prime}}$ be another dividing curve in $\widehat{C}$. Since, $\widehat{\phi}(\widehat{\beta}) \cap \widehat{\phi}(\widehat{\chi})=\varnothing, \widehat{A}$ and $\widehat{C}$ are on the same side of $\widehat{\phi}(\widehat{\beta})$; that is, $\widehat{\phi}(\widehat{\alpha})$ and $\widehat{\phi}\left(\widehat{\chi}^{\prime}\right)$ lie on the same side of $\widehat{\phi}(\widehat{\beta})$. Similarly, $\widehat{\phi}(\widehat{\beta})$ and $\widehat{\phi}\left(\widehat{\chi^{\prime}}\right)$ lie on the same side of $\widehat{\phi}(\widehat{\alpha})$. So the plane $\widehat{\phi}\left(\widehat{\chi^{\prime}}\right)$ is in the complementary component of $\widehat{\phi}(\widehat{\alpha}) \cup \widehat{\phi}(\widehat{\beta})$ that is bounded by both $\widehat{\phi}(\widehat{\alpha})$ and $\widehat{\phi}(\widehat{\beta})$. By the argument above, one of the planes $\widehat{\phi}(\widehat{\alpha}), \widehat{\phi}(\widehat{\beta})$ and $\widehat{\phi}\left(\widehat{\chi^{\prime}}\right)$ lies between the other two. So $\widehat{\phi}(\widehat{\alpha})$ and $\widehat{\phi}(\widehat{\beta})$ are on opposite sides of $\widehat{\phi}\left(\widehat{\chi^{\prime}}\right)$; that is, $\widehat{A}$ and $\widehat{B}$ are on opposite sides of $\widehat{\phi}\left(\widehat{\chi^{\prime}}\right)$.

By Condition (1) for a dividing curve, $\widehat{\phi}(\widehat{\alpha})$ is a properly imbedded plane. Consequently, the orientation of any $\widehat{K}$ chosen, as in Condition (2), to have intersection number with $\widehat{\phi}(\widehat{\alpha})$ equal to 1 can be used to define the positive side of $\widehat{\phi}(\widehat{\alpha})$. We say $\widehat{B}<\widehat{A}$ if $\widehat{B}$ is on the negative side of $\widehat{\phi}(\widehat{\alpha})$ and $\widehat{A}<\widehat{B}$ if $\widehat{B}$ is on the positive side of $\widehat{\phi}(\widehat{\alpha})$. Since $\widehat{A}$ and $\widehat{B}$ are nonseparable, $\widehat{B} \cap \widehat{\phi}(\widehat{\alpha})=$ $\varnothing$. It follows that either $\widehat{A}<\widehat{B}$ or $\widehat{B}<\widehat{A}$. Suppose $\widehat{A}<\widehat{B}$. There exists an $r>\max \left\{r_{\alpha}, r_{\beta}\right\}$ such for any leaf $\widehat{K}$ in $F_{0}(r)$ chosen sufficiently close to $\widehat{A} \cap P_{0}(r)$ and $\widehat{B} \cap P_{0}(r), \widehat{K}$ meets $\widehat{\phi}(\widehat{\alpha})$ and $\widehat{\phi}(\widehat{\beta})$, each with intersection number equal to 1 . So in this case, $\widehat{K}$ meets $\widehat{\phi}(\widehat{\alpha})$ transversely before meeting $\widehat{\phi}(\widehat{\beta})$. Consequently, $\widehat{A}$ is on the negative side of $\widehat{\phi}(\widehat{\beta})$. Clearly $\leq$ is reflexive, antisymmetric and transitive, so we have the following: 
Proposition 1.6. Let $F$ be a taut foliation with transverse flow $\phi$ such that every branching leaf of $\widehat{F}$ contains a dividing curve for $\widehat{\phi}$. Any collection of nonseparable leaves in $\widehat{F}$ is totally ordered by $\leq$.

Given a branching leaf $\widehat{A} \in \widehat{F}$, let $E_{-}(\widehat{A})$ denote the set consisting of $\widehat{A}$ and all leaves nonseparable from $\widehat{A}$ on the negative side.

We now show:

Theorem 1.7. Let $F$ be a taut foliation with transverse flow $\phi$ such that every branching leaf of $\widehat{F}$ contains a dividing curve for $\widehat{\phi}$. Given a branching leaf $\widehat{A} \in \widehat{F}$, suppose $E_{-}(\widehat{A})$ is order isomorphic to a subset of the integers. If there exists a covering translation d fixing $\widehat{A}$ such that $d(\widehat{A})<d(\widehat{B})$ whenever $\widehat{B} \in E_{-}(\widehat{A})$ and $\widehat{A}<\widehat{B}$, then each leaf in $E_{-}(\widehat{A})$ is left invariant by $d$. In particular, the quotient in $F$ of each leaf in $E_{-}(\widehat{A})$ contains a loop freely homotopic to a loop in A.

Proof. Assume $E_{-}(\widehat{A})$ is order isomorphic to a subset of the integers. We verify that any covering translation $d$ fixing $\widehat{A}$ preserves the order in $E_{-}(\widehat{A})$. By hypothesis, for any for $\widehat{B} \in E_{-}(\widehat{A})$, if $\widehat{A}<\widehat{B}$ then $d(\widehat{A})<d(\widehat{B})$. So it suffices to show that $d$ preserves betweeness for elements of $E_{-}(\widehat{A})$.

First note that $d$ preserves the foliation $\widehat{F}$ and the transverse flow $\widehat{\phi}$, but not necessarily the product foliation of $\widehat{M}$ by the family $\left\{P_{0}(r): r \in R\right\}$ of parallel imbedded planes. Now suppose $\widehat{B}_{1}, \widehat{B}_{2}$ and $\widehat{B}_{3}$ are leaves in $E_{-}(\widehat{A})$ such that $\widehat{B}_{1}-\widehat{B}_{2}-\widehat{B}_{3}$ (i.e., $\widehat{B}_{2}$ is between $\widehat{B}_{1}$ and $\widehat{B}_{3}$ ). Let $\widehat{\beta}_{1}, \widehat{\beta}_{2}$ and $\widehat{\beta}_{3}$ be dividing curves in $\widehat{B}_{1}, \widehat{B}_{2}$ and $\widehat{B}_{3}$ respectively. We may choose a curve $\widehat{\kappa}$ in a leaf on the negative side of $\widehat{A}$ so that the initial point of $\widehat{\kappa}$ flows, along an orbit of $\widehat{\phi}$, into $\widehat{\beta}_{1}$ and the terminal point of $\widehat{\kappa}$ flows into $\widehat{\beta}_{3}$. Clearly the ends of $\widehat{\kappa}$ lie on opposite sides of $\widehat{\phi}\left(\widehat{\beta}_{2}\right)$. Now the ends of $d(\widehat{\kappa})$ flow into $d\left(\widehat{\beta}_{1}\right)$ and $d\left(\widehat{\beta}_{3}\right)$ respectively and lie on opposite sides of $d\left(\widehat{\phi}\left(\widehat{\beta}_{2}\right)\right)$. It follows that $d\left(\widehat{B}_{1}\right)$ and $d\left(\widehat{B}_{3}\right)$ lie on opposite sides of $d\left(\widehat{\phi}\left(\widehat{\beta}_{2}\right)\right)$. Since $d\left(\widehat{B}_{1}\right), d\left(\widehat{B}_{2}\right)$ and $d\left(\widehat{B}_{3}\right)$ are nonseparable, they contain dividing curves $\widehat{\beta}_{1}^{\prime}, \widehat{\beta}_{2}^{\prime}$ and $\widehat{\beta}_{3}^{\prime}$ respectively and, by Proposition 1.5, precisely one of these leaves lies between the other two. Now $d\left(\widehat{B}_{1}\right)$ and $d\left(\widehat{B}_{2}\right)$ lie on the same side of $\widehat{\phi}\left(\beta_{3}^{\prime}\right)$ since $\widehat{\phi}\left(\beta_{3}^{\prime}\right) \cap d\left(\widehat{\phi}\left(\widehat{\beta}_{2}\right)\right)=\varnothing$. Likewise, $d\left(\widehat{B}_{2}\right)$ and $d\left(\widehat{B}_{3}\right)$ lie on the same side of $\widehat{\phi}\left(\beta_{1}^{\prime}\right)$. So $d\left(\widehat{B}_{1}\right)$ and $d\left(\widehat{B}_{3}\right)$ lie on opposite sides of $\widehat{\phi}\left(\beta_{2}^{\prime}\right)$; that is, $d\left(\widehat{B}_{1}\right)-d\left(\widehat{B}_{2}\right)-d\left(\widehat{B}_{3}\right)$. Therefore $d$ preserves betweeness for elements of $E_{-}(\widehat{A})$.

In general, finiteness of branching is dependent on the transverse flow $\widehat{\phi}$ since betweeness depends on this flow. However, for $\widehat{F}$ a stable Anosov foliation, finite branching as defined in $[\mathbf{F e} 4]$ is equivalent to finite branching with respect to the flow $\widehat{\phi}$ in the unstable direction. In this case, there is finite branching between any branching pair $(\widehat{A}, \widehat{B})[\mathbf{F e} 4$, Theorem $\mathrm{C}]$. 
A critical step in Fenley's proof is showing that $\widehat{B}$ is left invariant under any covering translation fixing $\widehat{A}[\mathbf{F e} 4$, Theorem D]. Each such covering translation fixes an orbit $\widehat{\alpha}$ of the Anosov flow and satisfies the conditions of Theorem 1.7 above. (This latter follows since $\widehat{\alpha}$ is a dividing curve and $\widehat{\phi}(\widehat{\alpha})$ covers a transversely orientable 2 -manifold $\phi(\alpha)$; in particular, $\phi(\alpha)$ is a leaf in the unstable Anosov foliation of $M$ so the covering translation preserves the transverse orientation of $\widehat{\phi}(\widehat{\alpha})$.) So we have that [Fe4, Theorem C] also implies $[\mathbf{F e} 4$, Theorem D].

\section{Branched surfaces and branching in the universal cover.}

In this section we use branched surfaces constructed from foliations. These branched surfaces are in the class of regular branched surfaces introduced by R. Williams $[\mathbf{W}]$ and are constructed according to a technique given in [Ch-Go]. We shall give a brief outline of this construction, including only those details necessary to understand the results which follow.

We begin with a foliation $F$, a flow $\phi$ transverse to $F$, and a generating set $\Delta=\left\{D_{i}\right\}$ of disjoint imbedded compact surfaces with boundary (which is finite if the ambient manifold $M$ is closed), satisfying the following general position requirements:

(i) each $D_{i}$ lies in a leaf of $F$ (hence is transverse to $\phi$ )

(ii) every orbit of $\phi$ meets the interior of some element of $\Delta$ in forward and backward time

(iii) for every $i_{0}$, the set of points in $B d y\left(D_{i_{0}}\right)$ whose orbit under $\phi$ meets $\cup B d y\left(D_{i}\right)$ before meeting $\operatorname{Uint}\left(D_{i}\right)$ is finite

(iv) any orbit of $\phi$ meets the boundary of at most two elements of $\Delta$.

We cut $M$ open along the interior of each element of $\Delta$ to obtain a submanifold $M^{*}$ which can be imbedded in $M$ so that its boundary contains $\cup B d y\left(D_{i}\right)$. This can be thought of as "blowing air" into the leaves of $F$ to create an air pocket at each element of the generating set. By requirement (ii) above, the restriction of $\phi$ to $M^{*}$ is a flow $\phi^{*}$ with the property that each orbit is homeomorphic to the unit interval. We next form a quotient space by identifying points that lie on the same orbit of $\phi^{*}$. That is, we take the quotient $M^{*} / \sim$, where $x \sim y$ if $x$ and $y$ lie on the same interval orbit of $\phi^{*}$. We may think of this as enlarging the components of $M-M^{*}$ until each interval orbit of $\phi^{*}$ is contracted to a point in $M$. The imbedded copy of the resulting quotient space is the branched surface $W$; it is called the branched surface corresponding to $(F, \phi, \Delta)$. By its construction, $W$ is transverse to $\phi$. The general position requirements for $\Delta$ imply that $W$ is a connected 2-dimensional complex with a set of charts defining local orientation preserving diffeomorphisms onto one of the models in the figure below, 
and such that the transition maps are smooth and preserve transverse orientation. (Each local model projects horizontally onto a vertical model of $\mathbf{R}^{2}$ so has a smooth structure induced by $T \mathbf{R}^{2}$ which pulls back to $W$.)
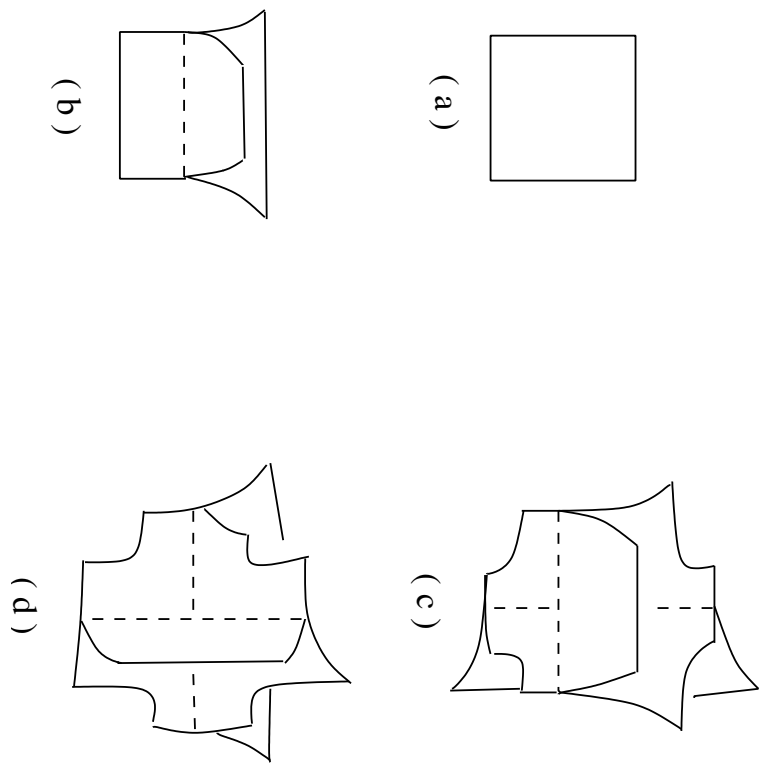

Figure 2.1.

So each branched surface obtained in the above fashion is a 2-manifold except on a small subset $\boldsymbol{\mu}$ called the maw. The set $\mu$ is a 1 -manifold except at isolated points called crossings where it intersects itself transversely. (There are only finitely many of these points when $M$ is closed.) Each component of $W-\mu$ is called a sector of $W$.

Note that if we thicken $W$ in the transverse direction to recover the interval orbits of $\phi^{*}$, we retrieve $M^{*}$ which, for that reason, we shall henceforth call $N(W)$, the neighborhood of $W$. The interval orbit of $\phi^{*}$ whose quotient is a point $x$ in $W$ will be referred to as the fiber of $N(W)$ over $x$. (See Figure 2.2.) 

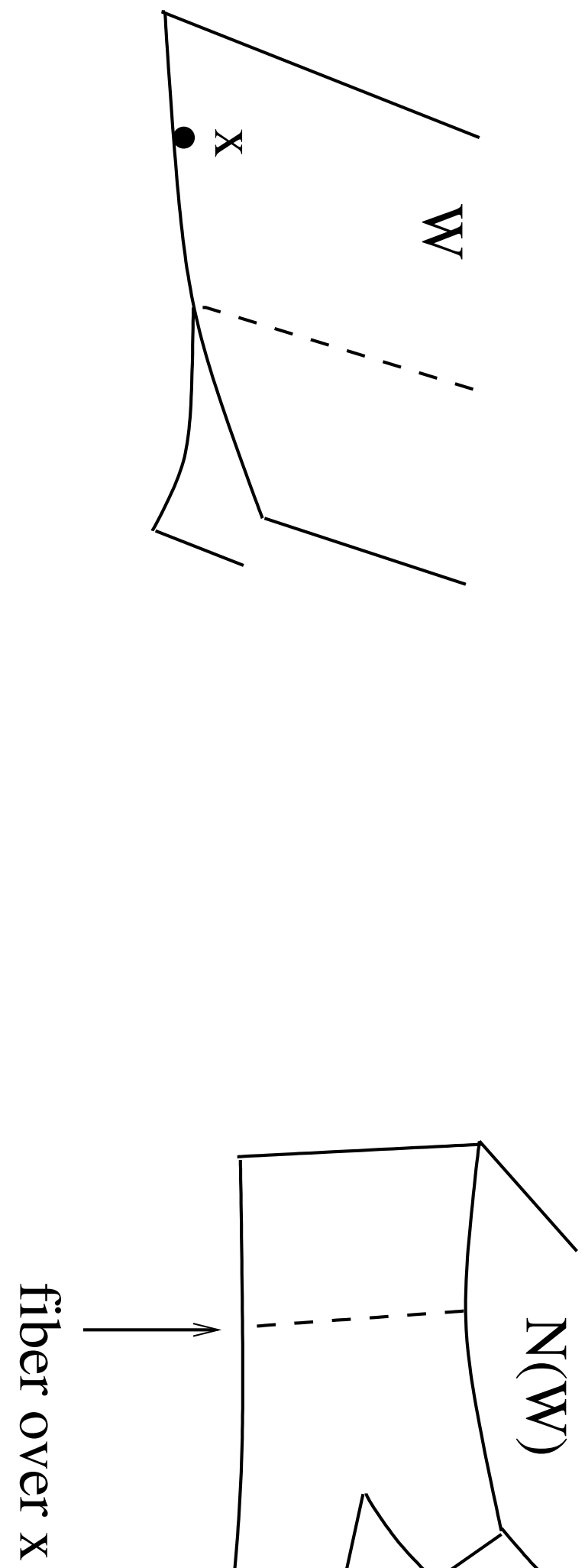


\section{Figure 2.2.}

A foliation $F$ clearly gives rise to a foliation of $N(W)$ with leaves transverse to the fibers of $N(W)$, which we shall also denote by $F$. Each leaf meeting the boundary of $N(W)$ contains the entire boundary component (these leaves can be thought of as leaves of the original foliation with air blown into them). That is, the leaves meeting the boundary of $N(W)$ are precisely the (cut-open) leaves of the original foliation containing elements of $\Delta$.

Throughout, $\pi: N(W) \rightarrow W$ will denote the quotient map which identifies points in the same fiber. We say the image $x$ of a point under this map is the projection of that point. Accordingly, we say points in preimage of $x$ lie over $x$.

In what follows, $W$ will be a branched surface constructed from a foliation $F$ of a closed manifold $M$, and $\widehat{W}$ will be the lift of $W$ to the universal cover $\widehat{M}$. In other words, if $W$ corresponds to $(F, \Delta, \phi)$, then $\widehat{W}$ is the branched surface corresponding to $(\widehat{F}, \widehat{\Delta}, \widehat{\phi})$, where $\widehat{\Delta}$ is the lift of $\Delta$ and $\widehat{\phi}$ is the lift of $\phi$ to the universal cover. The covering map $\rho_{M}$ of $M$ by $\widehat{M}$ acts on $N(\widehat{W})$ and induces a covering map $\rho: \widehat{W} \rightarrow W$ such that $\pi \circ \rho_{M}=\rho \circ \widehat{\pi}$. (Details are given in $[\mathbf{S h}]$.)

Suppose leaves $\widehat{A}$ and $\widehat{B}$ of $\widehat{F}$ are nonseparable on their negative sides; that is, there is a 1-parameter family $\left\{\widehat{K}_{n}\right\}$ of leaves, parameterized by $n \in I$ (where $I$ is the real interval $(0,1)$ ) which lie on the negative side of $\widehat{A}$ and $\widehat{B}$ and montonically approach $(\widehat{A}, \widehat{B})$ in the leaf space as $n \rightarrow 1$. (We assume points in the leaf space are ordered according to the transverse orientation of $\widehat{F}$.) When one considers what this behavior might yield in a branched surface, one is eventually led to the following type of picture: 


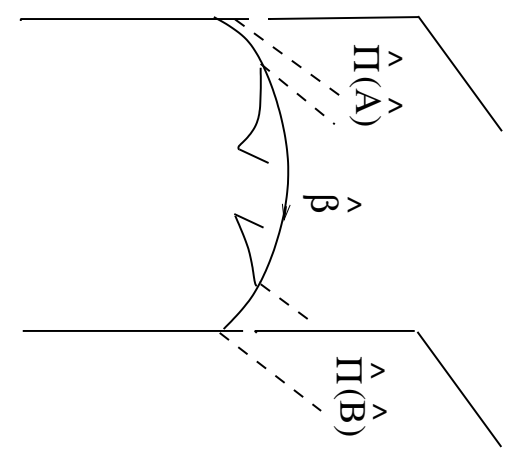

\section{Figure 2.3.}

More precisely, if $\widehat{F}$ contains a pair of leaves $\widehat{A}$ and $\widehat{B}$ that are nonseparable on their negative sides, then there is an arc $\widehat{\beta}$ in $\widehat{W}$ nowhere tangent to the maw $\widehat{\mu}$ (and containing no crossings of $\widehat{\mu}$ ) with its ends branching into the negative sides of $\widehat{\pi}(\widehat{A})$ and $\widehat{\pi}(\widehat{B})$ respectively [Go-Sh]. Such an arc $\widehat{\beta}$ can be chosen to lie in $\widehat{\pi}(\widehat{K})$ for some leaf $\widehat{K}$ of $\widehat{F}$ and is said to be a branching arc linking $\widehat{A}$ and $\widehat{B}$. (The branching arc $\widehat{\beta}$ covers a "branching arc" $\beta$ in $W$.) If there are no sectors branching into the positive side of $\widehat{\pi}(\widehat{K})$ which meet the interior of $\widehat{\beta}$, then $\widehat{\beta}$ and $\beta$ are said to be simple branching arcs. For example, $\widehat{\beta}$ in Figure 2.3 is a simple branching arc linking $\widehat{A}$ and $\widehat{B}$.

It is easy to see that any simple branching arc $\widehat{\beta}$ in $\widehat{W}$ corresponds to an arc in an element of $\widehat{\Delta}$ (generating $\widehat{W}$ ) with endpoints in the boundary of that generating surface. For such a branching arc, the leaf $\widehat{K}$ may be chosen so that it contains a curve $\widehat{\kappa}$ in $\partial N(\widehat{W})$ which lies over $\widehat{\beta}$ and begins in the interior of some fiber. When we collapse the components of $\widehat{M}-N(\widehat{W})$ to retrieve the original foliation $\widehat{F}$ of $\widehat{M}$, the image of $\widehat{\kappa}$ is contained in an element $\widehat{D}$ of $\widehat{\Delta}$, and its endpoints in $\partial \widehat{D}$ flow into $\widehat{A}$ and $\widehat{B}$ respectively before meeting $\widehat{\Delta}$ again. See Figure 2.4 . 


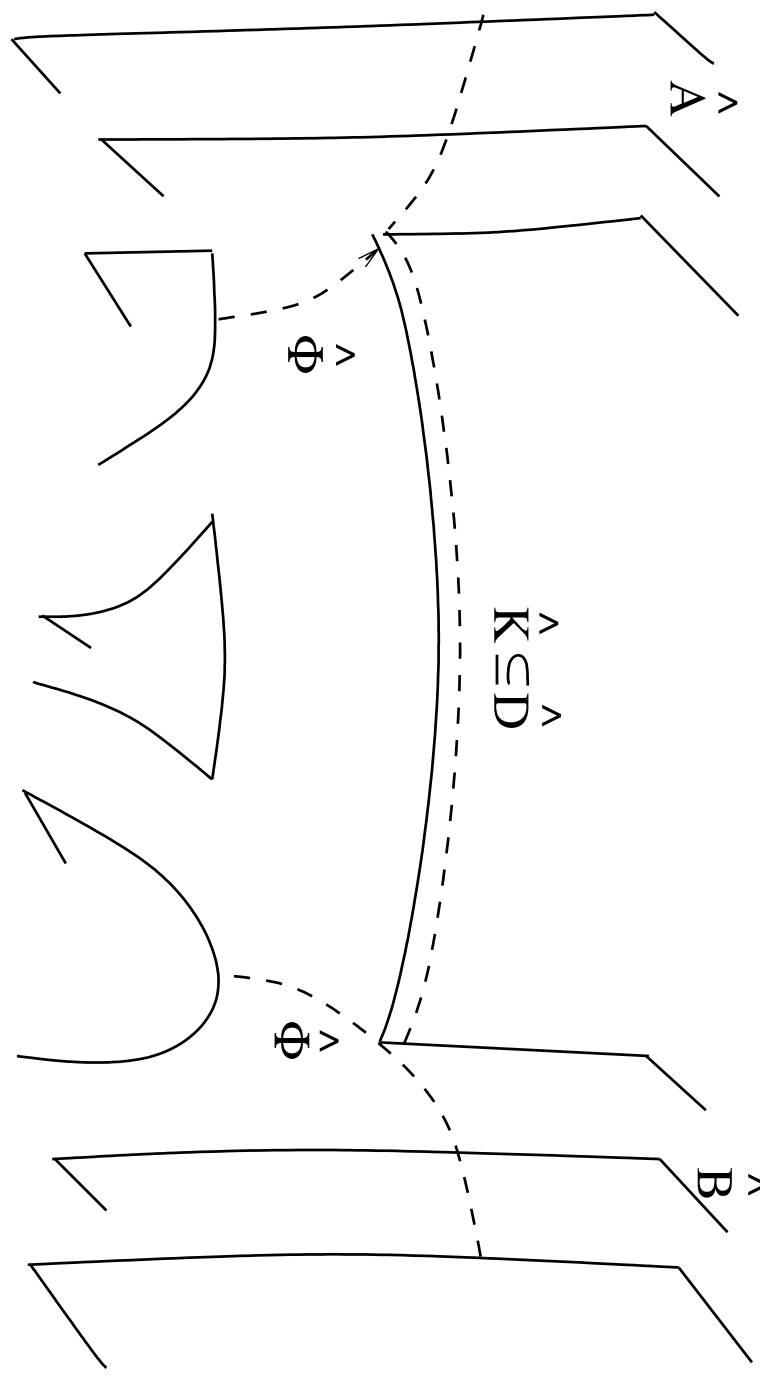

Figure 2.4.

Conversely, if for some branching arc $\widehat{\beta}$ with endpoints $\widehat{\beta}(0)$ and $\widehat{\beta}(1)$ respectively, the component $\widehat{C}$ of $\partial N(\widehat{W})$ meeting the interior of the fiber over $\widehat{\beta}(0)$ also meets the fiber over $\widehat{\beta}(1)$, then $\widehat{\beta}$ is simple.

Certain branching arcs in $\widehat{W}$ (and $W$ ) can be regarded as essentially the same. More precisely, the boundary of each sector $S$ is contained in the maw and can be partitioned by crossings into disjoint open arcs. Any two arcs whose interiors lie in $S$ are "equivalent" if the initial points of the arcs both lie in the interior of $S$ or in the same interval of the partition, and if the same condition holds for their terminal points. Any branching arc in $\widehat{W}(W)$ is a composition of finitely many arcs contained in sectors of $\widehat{W}$ 
( $W$ respectively). Accordingly, 2 branching arcs are equivalent if they are a composition of equivalent arcs. Indeed, if $\widehat{\beta}$ is a branching arc linking nonseparable leaves $\widehat{A}$ and $\widehat{B}$, and $\widehat{\beta}^{\prime}$ is equivalent to $\widehat{\beta}$, then $\widehat{\beta}^{\prime}$ is also a branching arc linking $\widehat{A}$ and $\widehat{B}$.

Now for each element of the finite set $\Delta$ (generating $W$ ), the corresponding component of $\partial(M-W)$ contains a finite number of sectors of $W$. It follows that the number of simple branching arcs in $W$ is finite up to equivalence.

We shall now use branched surfaces to prove Theorems 2.1 and 2.3.

Theorem 2.1. Let $F$ be a taut foliation with transverse flow $\phi$ such that every branching leaf of $\widehat{F}$ contains a dividing curve for $\widehat{\phi}$. Given a branching leaf $\widehat{A} \in \widehat{F}$, suppose $E_{-}(\widehat{A})$ is order isomorphic to the integers. There exists a branched surface $W$ carrying $F$ such that some simple branching arc in $W$ has at least 3 lifts linking distinct branching pairs in $E_{-}(\widehat{A})$ if and only if there are at most finitely many leaves of $F$ that lift to a leaf in $E_{-}(\widehat{A})$; in other words, the set $E_{-}(\widehat{A})$ is finite up to the action of covering translations. Proof. Suppose there exists a branched surface $W$ as above. Since $E_{-}(\widehat{A})$ is order isomorphic to the set $Z$ of integers, we may index the leaves in $E_{-}(\widehat{A})$ so that $E_{-}(\widehat{A})=\left\{\widehat{A}_{i}: i \in Z, \widehat{A}_{0}=\widehat{A}\right.$ and $\widehat{A}_{i}<\widehat{A}_{i+1}$ for all $\left.i\right\}$. It suffices to show there is a covering translation $d$ that maps some leaf $\widehat{A}_{i} \in E_{-}(\widehat{A})$ onto another leaf $\widehat{A}_{i+N}$ in $E_{-}(\widehat{A})$ such that $d\left(\widehat{A}_{i+N}\right) \neq \widehat{A}_{i}$. Then since by Theorem 1.7 the maps $d$ and $d^{-1}$ preserve betweeness, $\widehat{A}_{i+j+k N}=d^{k}\left(\widehat{A}_{i+j}\right)$ for every $0 \leq j \leq N$ and $k \in Z$; that is, $\rho_{M}\left(\widehat{A}_{i+j+k N}\right)=\rho_{M}\left(\widehat{A}_{i+j}\right)$.

By assumption, there is a simple branching arc $\beta$ in $W$ with 3 lifts $\widehat{\beta}_{i}, \widehat{\beta}_{j}$ and $\widehat{\beta}_{k}$ linking distinct branching pairs $\left(\widehat{A}_{i}, \widehat{A}_{i+a}\right),\left(\widehat{A}_{j}, \widehat{A}_{j+b}\right)$ and $\left(\widehat{A}_{k}, \widehat{A}_{k+c}\right)$ in $E_{-}(\widehat{A})$ respectively, such that $a b>0$. (By definition, $\widehat{\beta}_{i}, \widehat{\beta}_{j}$ and $\widehat{\beta}_{k}$ are contained in the projection of leaves $\widehat{K}_{i}, \widehat{K}_{j}$ and $\widehat{K}_{k}$ respectively into $\widehat{W}$.) In particular, some covering translate of $\widehat{\beta}_{j}$ is equal to $\widehat{\beta}_{i}$. So there exists a covering translation $d$ such that $\widehat{\beta}_{i}$ links $d\left(\widehat{A}_{j}\right)=\widehat{A}_{j}^{\prime}$ and $d\left(\widehat{A}_{j+b}\right)=\widehat{A}_{j+b}^{\prime}$. Furthermore, $\widehat{A}_{j}^{\prime}$ cannot cut the fiber over $\widehat{\beta}_{i}(1)$ (since $\widehat{A}_{j+b}^{\prime}$ meets this fiber). So since the leaf $\widehat{A}_{j}^{\prime}$ is on the positive side of $\widehat{K}_{i}$, the nonseparable points in the leaf space representing $\widehat{A}_{j}^{\prime}$ and $\widehat{A}_{j+b}^{\prime}$ cannot lie below those representing $\widehat{A}_{i}$ and $\widehat{A}_{i+a}$. Similarly, $\widehat{A}_{j}^{\prime}$ and $\widehat{A}_{j+b}^{\prime}$ cannot lie above $\widehat{A}_{i}$ and $\widehat{A}_{i+a}$ in the leaf space. It follows that $\widehat{A}_{i}=\widehat{A}_{j}^{\prime}$ and $\widehat{A}_{i+a}=\widehat{A}_{j+b}^{\prime}$. That is, $d\left(\widehat{A}_{j}\right)=\widehat{A}_{i}$ and $d\left(\widehat{A}_{j+b}\right)=\widehat{A}_{i+a}$. Since $a b>0, d\left(\widehat{A}_{i}\right) \neq A_{j}$. It follows that the set $E_{-}(\widehat{A})$ is finite up to the action of covering translations.

Conversely, suppose that $E_{-}(\widehat{A})$ is finite up to the action of covering translations. Let $W$ be a branched surface carrying $F$ and let $\widehat{W}$ be its lift to the universal cover. For each $i \in Z$, let $\widehat{\beta}_{i}$ be a branching arc in $\widehat{W}$ 
linking $\widehat{A}_{i}$ and $\widehat{A}_{i+1}$ which is contained in $\widehat{\pi}\left(\widehat{K}_{i}\right)$ for some leaf $\widehat{K}_{i}$ of $\widehat{F}$. We choose $\widehat{\beta}_{i}$ to be simple whenever possible. Substituting an equivalent arc if necessary, we may assume $\beta_{i}=\rho\left(\widehat{\beta}_{i}\right)$ is not a loop. We may also guarantee that if $\left(\widehat{A}_{j}, \widehat{A}_{j+1}\right)$ is a covering translate of the branching pair $\left(\widehat{A}_{i}, \widehat{A}_{i+1}\right)$, (i.e., $d\left(\widehat{A}_{i}\right)=\widehat{A}_{j}$ and $d\left(\widehat{A}_{i+1}\right)=\widehat{A}_{j+1}$ for some covering translation $\left.d\right)$, then $\beta_{i}=\beta_{j}$. So, up to the action of covering translations, the set $\left\{\widehat{\beta}_{i}: i \in Z\right\}$ is finite.

For some $i \in Z$, there exists infinitely many covering translates of $\widehat{\beta}_{i}$ linking distinct branching pairs in $E_{-}(\widehat{A})$. Suppose that $\widehat{\beta}_{i}$ is not simple. Let $\widehat{C}_{i}$ be the component of $\partial N(\widehat{W})$ meeting the interior of the fiber over $\widehat{\beta}_{i}(0)$. It follows that $\widehat{C}_{i}$ does not meet the fiber over $\widehat{\beta}_{i}(1)$. If the leaf of $\widehat{F}$ containing $\widehat{C}_{i}$ does not equal $\widehat{K}_{i}$, then it lies above $\widehat{K}_{i}$; in either case, this leaf lies below $\left(\widehat{A}_{i}, \widehat{A}_{i+1}\right)$ in the leaf space, hence meets the fiber over $\widehat{\beta}_{i}(1)$. Now $\widehat{C}_{i}$ corresponds to an element $\widehat{D}_{i}$ of $\widehat{\Delta}$. In particular, enlarging $\widehat{D}_{i} \in \widehat{\Delta}$ corresponds to splitting $N(\widehat{W})$ open further along a portion of the leaf containing $\widehat{C}_{i}$. We may therefore enlarge $\widehat{D}_{i}$ so that $\widehat{C}_{i}$ cuts the fiber over $\widehat{\beta}_{i}(1)$; we then obtain a curve $\widehat{\kappa}_{i}$ from the fiber over $\widehat{\beta}_{i}(0)$ to the fiber over $\widehat{\beta}_{i}(1)$ that is contained in the outwardly oriented section of $\widehat{C}_{i}$. See Figure 2.5. When we enlarge $\widehat{D}_{i}$ as above to include an additional portion of the leaf containing it, it is important to ensure that the corresponding portion of the quotient leaf in $N(W)$ does not meet the fibers over $\beta_{i}(0)$ and $\beta_{i}(1)$ more than once. This guarantees that there is no covering translate of $\widehat{D}_{i}$ cutting an orbit of $\widehat{\phi}$ from an end of $\widehat{\kappa}_{i}$ into $\widehat{A}_{i}$ or $\widehat{A}_{i+1}$. In particular, we ensure that in the process of modifying $\widehat{\Delta}$ (and hence $\Delta$ ) so that $\widehat{C}_{i}$ meets the fibers over both $\widehat{\beta}_{i}(0)$ and $\widehat{\beta}_{i}(1)$, a covering translate of $\widehat{C}_{i}$ does not destroy this property. 

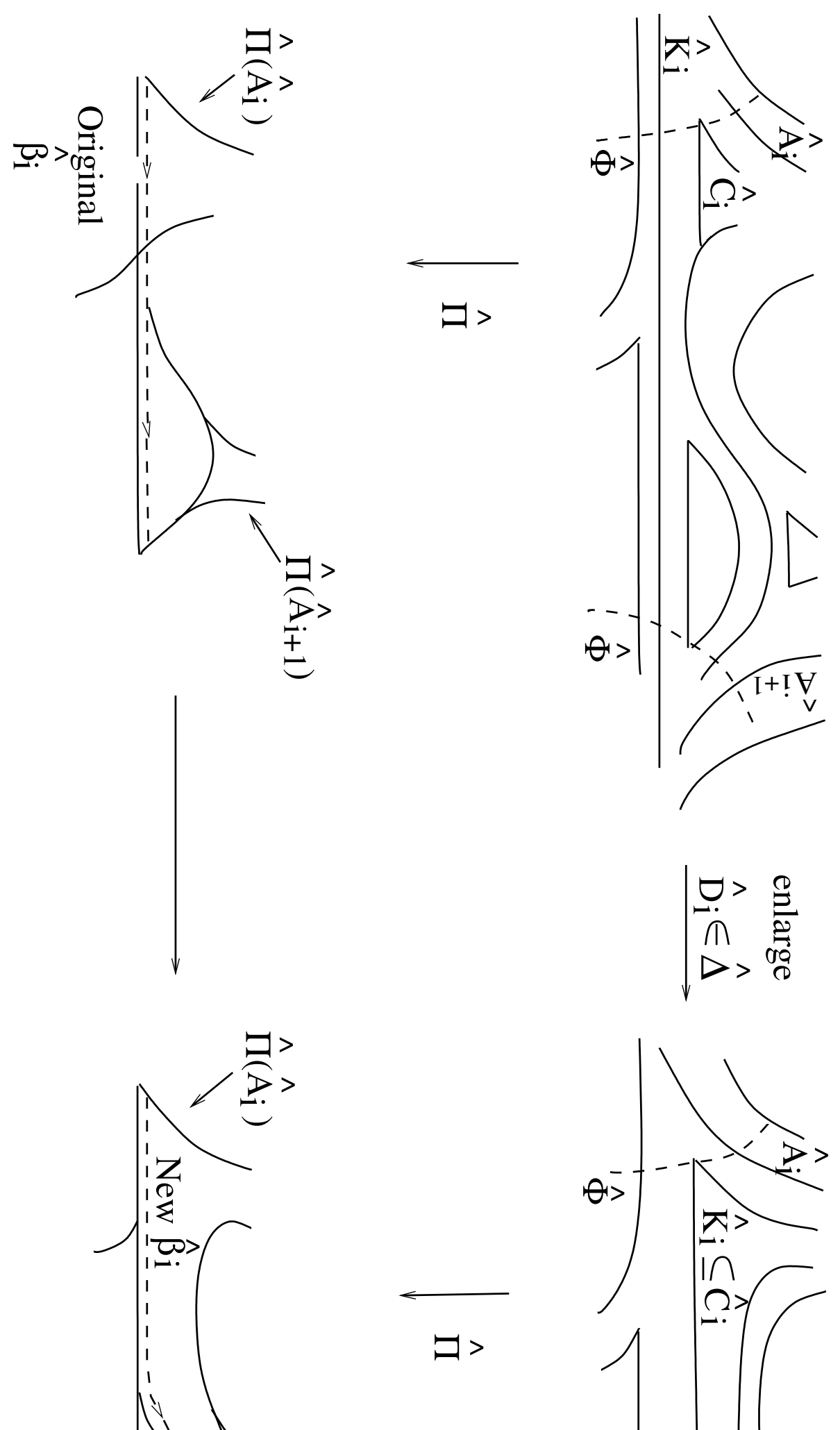


\section{Figure 2.5.}

Clearly when we modify $\widehat{W}$ in this manner to ensure $\widehat{\beta}_{i}$ is simple, we ensure all covering translates of $\widehat{\beta}_{i}$ are simple. So since there exists infinitely many covering translates of $\widehat{\beta}_{i}$ linking distinct branching pairs in $E_{-}(\widehat{A})$, we guarantee $\widehat{W}$ has the desired property.

If $F$ is Anosov, then for every leaf $\widehat{A}$ in the universal cover the set $E_{-}(\widehat{A})$ is order isomorphic to a subset of the integers $[\mathbf{F e} 4$, Theorem $\mathrm{C}]$. By $[\mathbf{F e} 4$, Theorem F], the conditions in Theorem 2.1 are satisfied by all Anosov foliations.

We now show the following:

Theorem 2.2. Let $F$ be a taut foliation with transverse flow $\phi$ such that each branching leaf of $\widehat{F}$ contains a dividing curve for $\widehat{\phi}$. For every leaf $\widehat{A}$ in $\widehat{F}$, the set $E_{-}(\widehat{A})$ is order isomorphic to a subset of the rational numbers $Q$.

Proof. We begin by taking a countable cover of $\widehat{M}$ by foliation boxes for $\widehat{F}$. We may associate any $\widehat{C} \in E_{-}(\widehat{A})$ with one of the boxes $U_{C}$ which it intersects. Now for every $\widehat{C}^{\prime} \in E_{-}(\widehat{A})$ such that $\widehat{C}^{\prime} \neq \widehat{C}, U_{C} \cap \widehat{C}^{\prime}=\varnothing$; in particular, $U_{C} \neq U_{C^{\prime}}$. Using Proposition 1.6, it follows that $E_{-}(\widehat{A})$ is a countable totally ordered set, hence it is order isomorphic to a subset of $Q$.

So for every $\widehat{B} \in E_{-}(\widehat{A})$, the subset of leaves in $E_{-}(\widehat{A})$ between $\widehat{A}$ and $\widehat{B}$ is order isomorphic to a subset of $Q$. Now consider the submanifold $N(\widehat{W})$ obtained during the construction of $\widehat{W}$ from $(\widehat{F}, \widehat{\phi}, \widehat{\Delta})$ and let $I_{A}$ and $I_{B}$ be fibers of $N(\widehat{W})$ through dividing curves $\widehat{\alpha}$ and $\widehat{\beta}$ in $\widehat{A}$ and $\widehat{B}$ respectively. We may choose an arc $\widehat{\gamma}_{1}$ in some leaf $\widehat{K}_{1}$ on the negative side of $\widehat{A}$ which begins in $I_{A}$, ends in $I_{B}$ and whose interior lies between $\widehat{\phi}(\widehat{\alpha})$ and $\phi(\widehat{\beta})$. Continuing inductively, we may choose a curve $\widehat{\gamma}_{n+1}$ in a leaf $\widehat{K}_{n+1}$ between $\widehat{K}_{n}$ and $\widehat{A}$ with the property that any fiber through an element of $E_{-}(\widehat{A})$ which is met by $\widehat{\gamma}_{n}$ is also met by $\widehat{\gamma}_{n+1}$. In this manner we obtain a sequence of $\operatorname{arcs}\left\{\widehat{\gamma}_{n}\right\}$. By carefully choosing the leaves in the sequence $\left\{\widehat{K}_{n}\right\}$, we may guarantee that these leaves converge monotonically to $\widehat{A}$ in the leaf space. We may also ensure that the interior of each arc $\widehat{\gamma}_{n}$ in this sequence lies between $\widehat{\phi}(\widehat{\alpha})$ and $\widehat{\phi}(\widehat{\beta})$ and that if it meets fibers through some $\widehat{C} \in E_{-}(\widehat{A})$ at points $x$ and $y$, then each point of $\widehat{\gamma}_{n}$ between $x$ and $y$ intersects a fiber through $\widehat{C}$. Now since $E_{-}(\widehat{A})$ is order isomorphic to a subset of the rational numbers $Q$, it is countable. So we may assume $\left\{\widehat{\gamma}_{n}\right\}$ has been chosen so that for every $\widehat{C} \in E_{-}(\widehat{A})$, there is a positive integer $n$ such that $\widehat{\gamma}_{n}$ meets a fiber through $\widehat{C}$. 
Now if some $\widehat{\gamma}_{n}$ meets fibers through a leaf $\widehat{C} \in E_{-}(\widehat{A})$, there is a first point and a last point along $\widehat{\gamma}_{n}$ for which this is the case. So if $\widehat{C} \neq \widehat{A}$ or $\widehat{B}$, it follows that $\widehat{\gamma}_{n}$ yields a curve $\widehat{\pi}\left(\widehat{\gamma}_{n}\right)$ in $\widehat{W}$ with the image $\widehat{\pi}(\widehat{C})$ of $\widehat{C}$ branching from it, as shown in Figure 2.6. That is, $\widehat{\pi}\left(\widehat{\gamma}_{n}\right)$ contains 2 arcs (indicated by the dashed segments), each with an end branching into the negative side of $\widehat{\pi}(\widehat{C})$ whose interiors do not intersect $\widehat{\pi}(\widehat{C})$. Clearly $\widehat{\pi}\left(\widehat{C}^{\prime}\right)$ for any other $\widehat{C}^{\prime} \in E_{-}(\widehat{A})$ does not meet $\widehat{\pi}\left(\widehat{\gamma}_{n}\right)$ between these two arcs. 


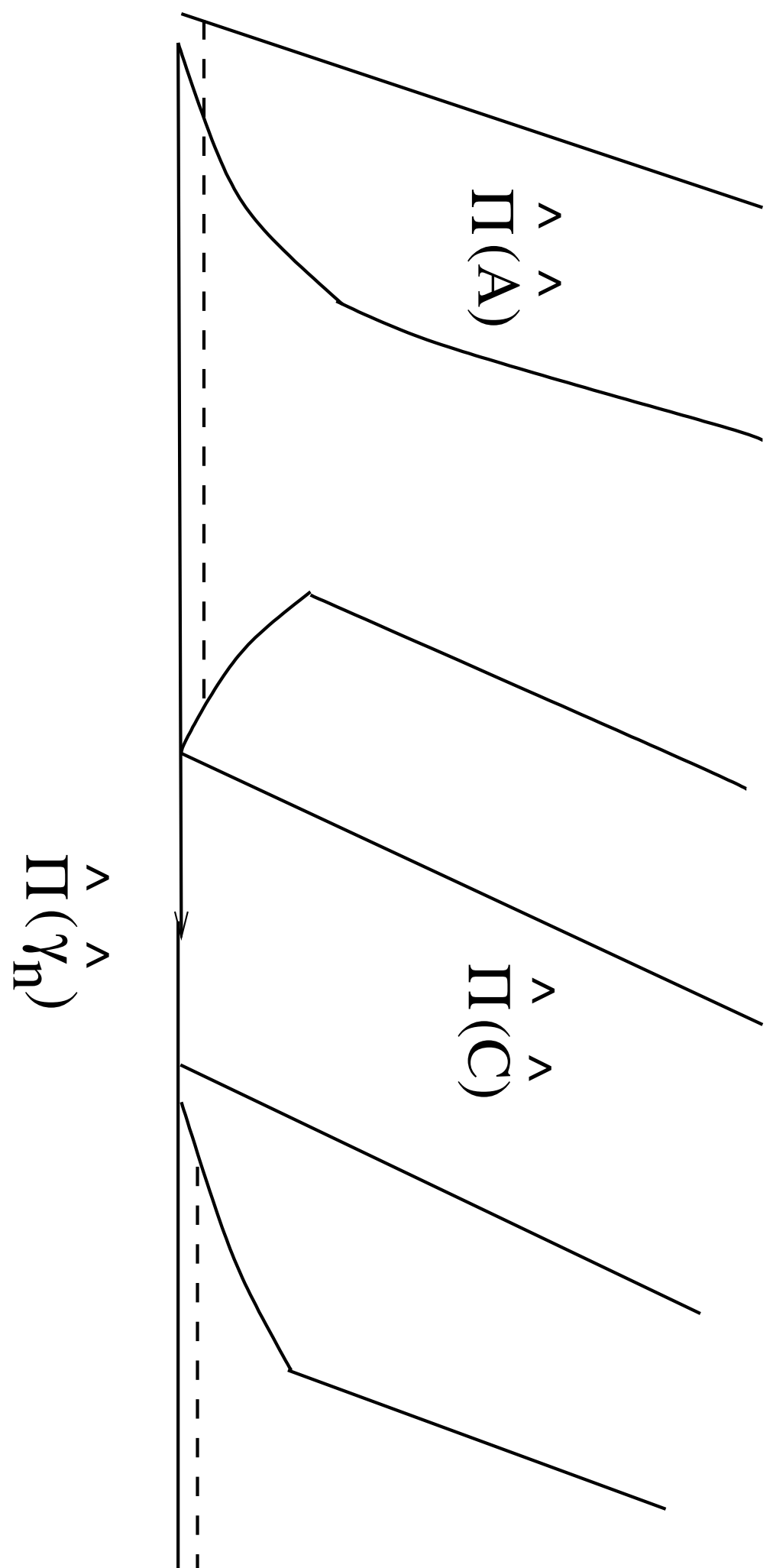




\section{Figure 2.6.}

For every $n$, the subset $\Sigma_{n}=\left\{\widehat{C} \in E_{-}(\widehat{A}): \widehat{\pi}(\widehat{C}) \cap \widehat{\pi}\left(\widehat{\gamma}_{n}\right) \neq \varnothing\right\}$ is finite. For suppose that, on the contrary, $\Sigma_{n}$ is infinite for some $n$. Then there exists infinitely many branching of $\widehat{W}$ along $\widehat{\pi}\left(\widehat{\gamma}_{n}\right)$ and these branchings accumulate in the lift of some evenly covered neighborhood $U$ of $W$. It follows that $U$ contains infinitely many branching of $W$ along some component of $\pi\left(\gamma_{n}\right) \cap U$, which is impossible under our construction of $W$.

Now given $\widehat{C}$ in $E_{-}(\widehat{A})$ between $\widehat{A}$ and $\widehat{B}$, there exists an integer $N_{C}>0$ such that $\widehat{C} \in \Sigma_{n}$ for all $n>N_{C}$ (see Figure 2.7). Moreover, for every $n$ there is a homeomorphism from $\widehat{\pi}\left(\widehat{\gamma}_{n}\right)$ onto $[0,1]$. These homeomorphisms can be chosen so that for any $\widehat{C} \in \Sigma_{n}$, the mapping on $\widehat{\pi}\left(\widehat{\gamma}_{n}\right)$ takes $\widehat{\pi}(\widehat{C}) \cap \widehat{\pi}\left(\widehat{\gamma}_{n}\right)$ to the same subinterval for all $n>N_{C}$. 


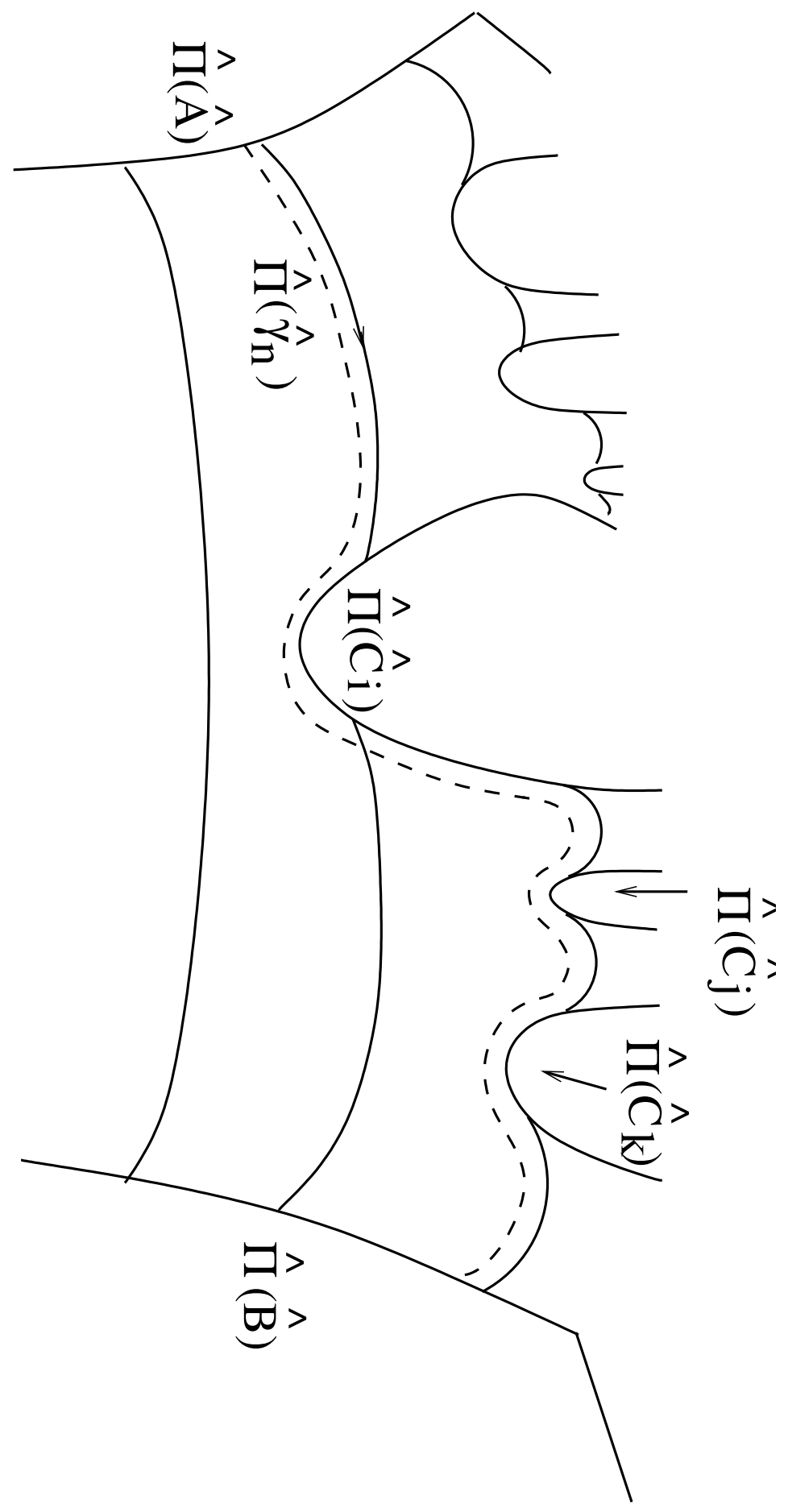




\section{Figure 2.7.}

In particular, we have:

Theorem 2.3. Let $F$ be a taut foliation with transverse flow $\phi$ such that each branching leaf of $\widehat{F}$ contains a dividing curve for $\widehat{\phi}$. For any positively branching pair $\widehat{A}$ and $\widehat{B}$ in $\widehat{F}$, there is an order preserving map $f$ assigning each element of $E_{-}(\widehat{A})$ between $\widehat{A}$ and $\widehat{B}$ to a connected component of $[0,1]-$ $\Sigma, \Sigma$ a Cantor set, and such that $f(\widehat{A})=\{0\}$ and $f(\widehat{B})=\{1\}$. Moreover, for any finite subset $\left\{\widehat{C}_{1}, \ldots, \widehat{C}_{n}\right\}$ of leaves between $\widehat{A}$ and $\widehat{B}$, there is an imbedded copy $\widehat{\gamma}$ of $[0,1]$ in $\widehat{W}$ intersecting each $\widehat{\pi}\left(\widehat{C}_{i}\right)$ in $f\left(\widehat{C}_{i}\right)$ with the property that for every $\widehat{C}^{\prime} \in E_{-}(\widehat{A})$, either $\widehat{\pi}\left(\widehat{C}^{\prime}\right) \cap \widehat{\gamma}=\varnothing$ or $\widehat{\pi}\left(\widehat{C}^{\prime}\right) \cap \widehat{\gamma}=$ $f\left(\widehat{C}^{\prime}\right)$.

According to Theorem 1.7, $E_{-}(\widehat{A})$ is order isomorphic to a subset of the integers only when every $\widehat{B} \in E_{-}(\widehat{A})$ is left invariant by certain covering translations fixing $\widehat{A}$. To check that the latter is the case, it is sufficient to verify that the corresponding surface $\widehat{\pi}(\widehat{B})$ in the combinatorial object $\widehat{W}$ is left invariant by the corresponding covering translation of $\widehat{W}$. Before proving this (in Proposition 2.5) we review the relationship between covering translations of $\widehat{M}$ and "covering translations of $\widehat{W}$ ".

Definition 2.4. A diffeomorphism $D_{\widehat{W}}$ of $\widehat{W}$ is called a covering translation of $\widehat{W}$ if for some covering map $p: \widehat{W} \rightarrow W, p \circ D_{\widehat{W}}=p$.

Suppose $D_{\widehat{M}}$ is a covering translation of $\widehat{M}$. Then $D_{\widehat{M}}$ preserves the transverse flow $\widehat{\phi}$ (i.e., maps orbits to orbits). Since the generating set for $\widehat{W}$ is the lift of the generating set for $W$, it is also preserved by $D_{\widehat{M}}$. Therefore, $D_{\widehat{M}}$ maps each fiber of $N(\widehat{W})$ onto another fiber. Consequently, when we collapse fibers of $N(\widehat{W}), D_{\widehat{M}}$ induces a covering translation $D_{\widehat{W}}$ of $\widehat{W}$; in other words, on $N(\widehat{W}), \widehat{\pi} \circ D_{\widehat{M}}=D_{\widehat{W}} \circ \widehat{\pi}$.

Conversely, suppose $D_{\widehat{W}}$ is a nontrivial covering translation of $\widehat{W}$. For any $\widehat{x}$ in $\widehat{W}$, if $D_{\widehat{W}}(\widehat{x})=\widehat{y}$, then $x=p(\widehat{x})=p \circ D_{\widehat{W}}(\widehat{x})=p(\widehat{y})=y$. We may define a homeomorphism from the fiber over $\widehat{x}$ onto the fiber over $\widehat{y}$ which covers the identity map on the fiber over $x$ (under the covering map $p_{M}$ of $M$ corresponding to $p$ ). In this way, $D_{\widehat{W}}$ gives rise to a covering translation $D_{\widehat{M}}$ of the manifold $N(\widehat{W})$ and, after we collapse the complement of $N(\widehat{W})$ in $\widehat{M}, D_{\widehat{M}}$ is a covering translation of $\widehat{M}$. In particular, $\widehat{\pi} \circ D_{\widehat{M}}=D_{\widehat{W}} \circ \widehat{\pi}$.

Proposition 2.5. A branching leaf $\widehat{A}$ is left invariant under a covering translation $D_{\widehat{M}}$ of $M$ if and only if $\widehat{\pi}(\widehat{A})$ is left invariant under the corresponding covering translation $D_{\widehat{W}}$ of $\widehat{W}$. 
Proof. Without loss of generality, assume $\widehat{A}$ is a positively branching leaf. Clearly, if $\widehat{A}$ is left invariant under a covering translation $D_{\widehat{M}}$ of $\widehat{M}$, then $\widehat{\pi}(\widehat{A})$ is left invariant under the induced covering translation $D_{\widehat{W}}$ of $\widehat{W}$. So suppose $\widehat{A}$ is not left invariant by $D_{\widehat{M}}$ yet the induced translation $D_{\widehat{W}}$ leaves $\widehat{\pi}(\widehat{A})$ invariant. Then there exists a positively branching leaf $\widehat{A}^{\prime} \neq \widehat{A}$ such that $D_{\widehat{M}}(\widehat{A})=\widehat{A}^{\prime}$ and $\widehat{\pi}(\widehat{A})=\widehat{\pi}\left(\widehat{A}^{\prime}\right)$. It follows that $\widehat{A}$ and $\widehat{A}^{\prime}$ are separable (since fibers meeting both leaves are contained in orbits of the transverse flow). Without loss of generality, assume $\widehat{A}^{\prime}$ is on the negative side of $\widehat{A}$. By assumption, $\widehat{A}$ is a positively branching leaf, so there exists a leaf $\widehat{B} \in E_{-}(\widehat{A})$ and a leaf $\widehat{K}$ on the negative side of $\widehat{A}$ and $\widehat{B}$ which meets a fiber though $\widehat{A}$ and a fiber through $\widehat{B}$. Since $\widehat{A}^{\prime}$ and $\widehat{A}$ meet the same fibers of $N(\widehat{W}), \widehat{A}^{\prime}$ cannot meet any fiber over $\widehat{B}$. So for $\widehat{K}$ sufficiently close to $\widehat{A}$ and $\widehat{B}$, the leaf $\widehat{A}^{\prime}$ is on the negative side of $\widehat{K}$, and $\widehat{\pi}\left(\widehat{A}^{\prime}\right)$ branches away from $\widehat{\pi}(\widehat{K}) \cap \widehat{\pi}(\widehat{A})$. This means $\widehat{\pi}(\widehat{A})$ contains all sectors whose boundaries include the segment of the maw at this branching (see Figure 2.8), a contradiction since $F$ taut implies $\widehat{\pi}$ is injective on $\widehat{A}$. 


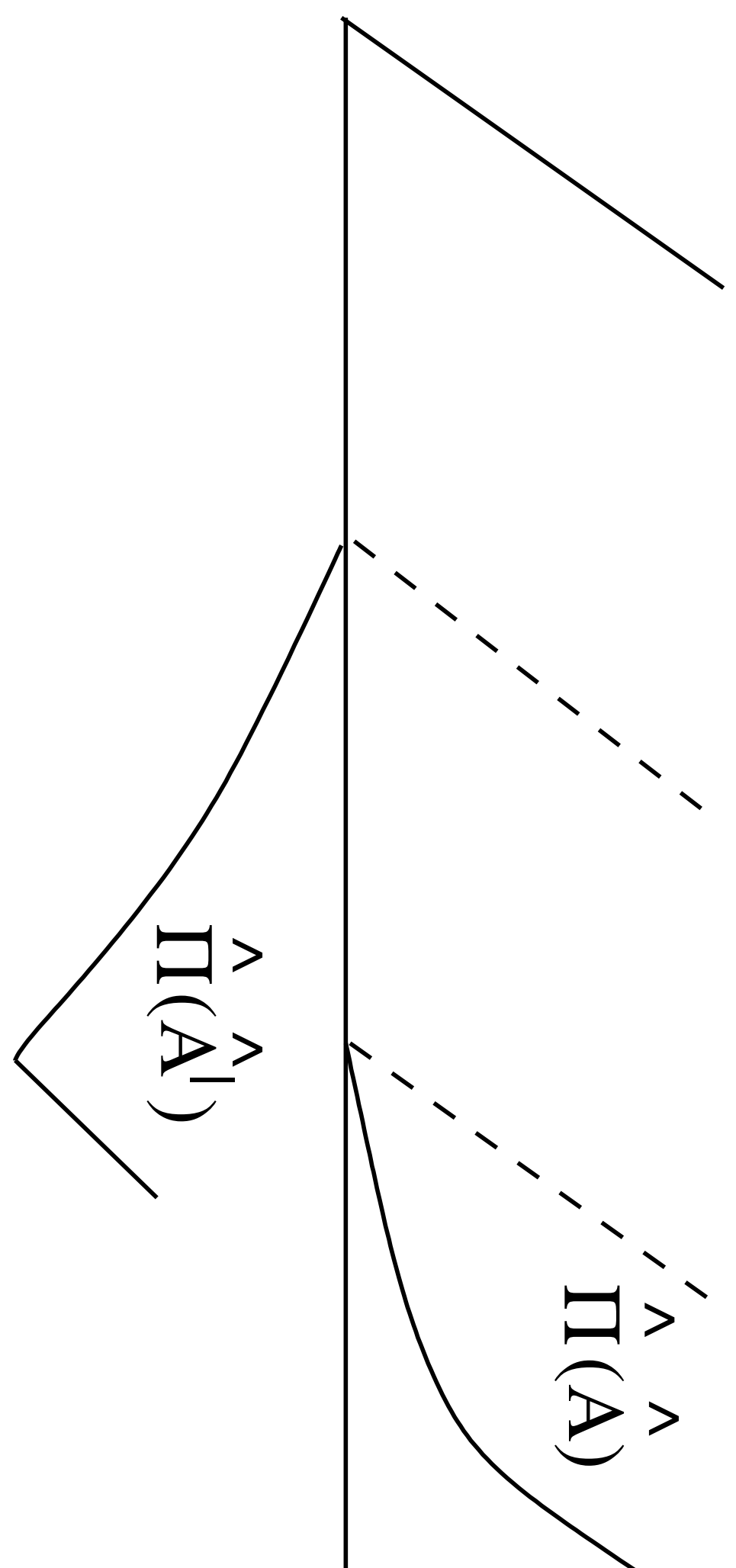




\section{Figure 2.8.}

\section{References}

[An] D.V. Anosov, Geodesic flows on closed Riemannian manifolds with negative curvature, Proc. Steklov Inst. Math., 90 (1969), MR 39 \#3527, Zbl 176.19101.

[Ba1] T. Barbot, Geometrie transverse des flots d'Anosov, Thesis, Ecole Norm. Sup., Lyon, 1992.

[Ba2] Caracterisation des flots d'Anosov en dimension 3 par leurs feuilletages faibles, Erg. Theory Dynam. Systems, 15 (1995), 247-470, MR 96d:58100.

[Bo-La] C. Bonatti and R. Langevin, Un example de flot D'Anosov transitif transverse a un tore et non conjugue a une suspension, Erg. Theory Dynam. Systems, 14 (1994), 633-643, MR 95j:58129, Zbl 826.58026.

[Ch-Go] J. Christy and S. Goodman, Branched surfaces transverse to codimension one foliations, preprint.

[Fe1] S. Fenley, Anosov flows in 3-manifolds, Ann. of Math., 139 (1994), 79-115, MR 94m:58162, Zbl 796.58039.

[Fe2] Continuous extension of Anosov foliations in 3-manifolds with negatively curve fundamental group, Pacific Journal of Math., 186(2) (1998), 201-216, MR 99k:58135.

[Fe3] - Homotopic indivisibility of closed orbits of Anosov flows, Math. Zeit., 225 (1997), 289-294, MR 98f:58152, Zbl 936.37007.

[Fe4] The structure of branching in the Anosov flows of 3-manifolds, Comment. Math. Helv., 73(2) (1998), 259-297, MR 99a:58123.

[Fl-Oe] W. Floyd and U. Oertel, Incompressible surfaces via branched surfaces, Topology, 23 (1984), 117-125, MR 85a:57007, Zbl 524.57008.

[Fr-Wi] J. Franks and R. Williams, Anomalous Anosov flows, Common Theory of Dynamical Systems, Lecture notes in Mathematics, 819, Springer, Berlin, (1980), 158-179, MR 82e:58078, Zbl 463.58021.

[Ga1] D. Gabai, Foliations and the topology of 3-manifolds, Journal of Differential Geometry, 18 (1983), 445-503, MR 86a:57009, Zbl 533.57013.

[Ga2] Foliations and the topology of 3-manifolds II, Journal of Differential Geometry, 26 (1987), 461-478, MR 89a:57014a, Zbl 627.57012.

[Ga3] Foliations and the topology of 3-manifolds III, Journal of Differential Geometry, 26 (1987), 479-536, MR 89a:57014b, Zbl 639.57008.

[Ga-Ka] D. Gabai and W. Kazez, Homotopy, isotopy and genuine laminations of 3manifolds, Studies in Advanced Mathematics: Geometric Topology, 2 (1993), 123-138, MR 98k:57026, Zbl 893.57012.

[Gh] E. Ghys, Flots d'Anosov sur les 3-varietes fibres en cercles, Erg. Theory Dynam. Systems, 4 (1984), 67-80, MR 86b:58098, Zbl 538.58028.

[Go-Sh] S. Goodman and S. Shields, A condition for the stability of $R$-covered on foliatons of 3-manifolds, Trans. Amer. Math. Soc., 352(9) (2000), 4051-4065, MR 2000m:57042. 
[Im] H. Imanishi, On the theorem of Denjoy-Sacksteder for codimension one foliations without holonomy, J. Math. Kyoto Univ., 14 (1974), 607-634, MR 51 \#4270, Zbl 296.57006.

[NO] S.P. Novikov, Topology of foliations, Trans. Moscow Math. Soc., 14 (1965), 248278 (Russsian); A.M.S. translation, (1967), 268-304, MR 34 \#824, Zbl 247.57006.

[Pa] F. Palmeira, Open manifolds foliated by planes, Annals of Math., 107 (1978), 109-131, MR 58 \#18490, Zbl 382.57010.

[Pl1] J. Plante, Anosov flows, transversely affine foliations and a conjecture of Verjovsky, J. London Math. Soc., 23(2) (1981), 359-362, MR 82g:58069, Zbl 465.58020.

[Pl2] S Solvable groups acting on the line, Trans. Amer. Math. Soc., 278 (1983), 401-414, MR 85b:57048, Zbl 569.57012.

[Pl3] _ Diffeomorphisms without periodic points, Proc. AMS, 88 (1983), 716718, MR 84j:58102, Zbl 519.58038.

[R] R. Roussarie, Plongements dans les varietes feuilletees et classification de feulletages sans holonomie, I.H.E.S. Sci. Publ. Math., 43 (1973), 101-142, MR 50 \#11268.

[Sh] S. Shields, The stability of foliations of orientable 3-manifolds covered by a product, Trans. Amer. Math. Soc., 348 (1996), 4653-4671, MR 97a:57019, Zbl 869.57003.

[So] V. Solodov, On the universal cover of Anosov flows, research announcement.

[Sp] M. Spivak, A Comprehensive Introduction to Differential Geometry, Volume 1, Publish or Perish Press, 1970, MR 82g:53003a, Zbl 439.53001.

[V] A. Verjovsky, Codimension one Anosov flows, Bol. Soc. Mat. Mexicana, 19 (1974), 49-77, MR 55 \#4282, Zbl 323.58014.

[W] R.F. Williams, Expanding attractors, Institut des Hautes Etudes Scientifiques; Publications Mathematiques, 43 (1973), 473-487, MR 50 \#1289, Zbl 279.58013.

Received March 9, 1999 and revised January 4, 2001.

Department of Mathematics, Maybank Hall

University of ChaRleston

Charleston, SC 29424-0001

E-mail address: shieldss@cofc.edu 